\title{
VRK1 is a Paralog Synthetic Lethal Target in VRK2-methylated Glioblastoma
}

Julie A. Shields ${ }^{1}$, Samuel R. Meier ${ }^{1}$, Madhavi Bandi ${ }^{1}$, Maria Dam Ferdinez ${ }^{1}$, Justin L. Engel $^{1}$, Erin E. Mulkearns-Hubert ${ }^{2}$, Nicole Hajdari ${ }^{2}$, Kelly Mitchell ${ }^{2}$, Wenhai Zhang ${ }^{1}$, Shan-chuan Zhao ${ }^{1}$, Minjie Zhang ${ }^{1}$, Robert Tjin Tham Sjin ${ }^{1}$, Erik Wilker ${ }^{1}$, Justin D. Lathia $^{2}$, Jannik N. Andersen ${ }^{1}$, Yingnan Chen ${ }^{1}$, Fang $\mathrm{Li}^{1}$, Barbara Weber ${ }^{1}$, Alan Huang ${ }^{1}$ and Natasha Emmanuel ${ }^{1, *}$

${ }^{1}$ Tango Therapeutics, Cambridge MA

${ }^{2}$ Cleveland Clinic, Cleveland $\mathrm{OH}$

*Corresponding author: nemmanuel@tangotx.com

Running title: VRK1 is a synthetic lethal target in VRK2-methylated GBM

Keywords: VRK1, VRK2, glioblastoma, synthetic lethal, kinase, paralog

Corresponding Author: Natasha Emmanuel, Tango Therapeutics, 100 Binney Street, Suite 700, Cambridge MA 02114, +1-857-320-4900, nemmanuel@tangotx.com

\section{Disclosures}

J.A.S., S.R.M., M.B., M.D.F., J.L.E., W.Z., S.Z., M.Z., R.T.T.S., A.H., J.N.A, E.W., Y.C., F.L. and N.E are employees and shareholders of Tango Therapeutics. 


\section{ABSTRACT}

Synthetic lethality - a genetic interaction that results in cell death when two genetic deficiencies co-occur but not when either deficiency occurs alone - can be co-opted for cancer therapeutics. A pair of paralog genes is among the most straightforward synthetic lethal interaction by virtue of their redundant functions. Here we demonstrate a paralog-based synthetic lethality by targeting Vaccinia-Related Kinase 1 (VRK1) in Vaccinia-Related Kinase 2 (VRK2)-methylated glioblastoma (GBM). VRK2 is silenced by promoter methylation in approximately two-thirds of GBM, an aggressive cancer with few available targeted therapies. Genetic knockdown of VRK1 in VRK2-null or VRK2methylated cells results in decreased activity of the downstream substrate Barrier to Autointegration Factor (BAF), a regulator of post-mitotic nuclear envelope formation. VRK1 knockdown, and thus reduced BAF activity, causes nuclear lobulation, blebbing and micronucleation, which subsequently results in G2/M arrest and DNA damage. The VRK1-VRK2 synthetic lethal interaction is dependent on VRK1 kinase activity and is rescued by ectopic VRK2 expression. Knockdown of VRK1 leads to robust tumor growth inhibition in VRK2-methylated GBM xenografts. These results indicate that inhibiting VRK1 kinase activity could be a viable therapeutic strategy in VRK2methylated GBM.

\section{INTRODUCTION}

Recent years have witnessed a profound interest in targeting vulnerabilities in cancer stemming from synthetic lethal interactions - an approach to cancer treatment that specifically targets cancer cells while sparing normal healthy cells, thus increasing the therapeutic index of the therapeutic agent (1-3). The success of poly (ADP-ribose) 
polymerase 1 (PARP-1) inhibitors which are synthetic lethal with BRCA1 and BRCA2 mutations as well as other "BRCA-like" defects in homologous recombination demonstrated the potential of this therapeutic approach (4), and a subsequent largescale cancer dependency map (DepMap) resulted in discovery of additional novel synthetic lethal relationships (5-8). Using our proprietary bioinformatics pipeline, referred to as Tango Cancer Dependency Map (TANDEM), we analyzed public functional genomics data including the Cancer Cell Line Encyclopedia (CCLE) and identified one such novel paralog synthetic lethality, wherein VRK2-methylated GBM cell lines w19ere sensitive to loss of VRK1.

VRKs are a family of serine/threonine kinases that play a role in regulating transcription factors, chromatin remodeling, nuclear envelope formation, and cell cycle progression (9). There are three members of the VRK family - VRK1, VRK2 and a pseudokinase VRK3. VRK1 is found in both the nucleus and cytosol, and VRK2 localizes to the endomembrane of the endoplasmic reticulum and nuclear envelope (9). Functionally, VRK1 phosphorylates multiple substrates involved in both cell cycle progression and cell cycle arrest. Specifically, in response to mitogenic stimuli, VRK1 phosphorylates histones $\mathrm{H} 3$ and $\mathrm{H} 2 \mathrm{AX}$ to facilitate chromatin remodeling, transcription factors ATF2, CREB, Sox2, and farnesoid X nuclear receptor HR1H4 to promote cell cycle progression, and BAF to regulate nuclear envelope formation $(10,11)$. In response to stress signals, such as DNA damage, VRK1 phosphorylates p53, c-Jun, and 53BP1 to initiate cell cycle arrest for DNA damage repair $(10,11)$. The functional role of VRK2 is less clear, however, it has been reported to downregulate apoptosis via direct interaction with anti-apoptotic protein $\mathrm{Bcl}-\mathrm{xL}$ and by downregulating pro-apoptotic Bax 
(12). p53 and BAF have also been reported as substrates for VRK2 $(13,14)$. Additionally, cells with low expression of VRK2 have shown enhanced sensitivity to chemotherapeutics (12).

The World Health Organization (WHO) classifies adult, diffuse gliomas into three types - astrocytoma, oligodendroglioma, and GBM (15). WHO further classifies gliomas based on isocitrate dehydrogenase (IDH) mutation status, thus allowing tumors to be classified considering both phenotype and genotype. GBM is the most common primary malignant brain tumor and is uniformly fatal due to minimal success with current and novel therapies (16). Approximately $90 \%$ of GBM is IDH-wildtype, typically primary GBM, and these patients have lower overall survival compared to IDH-mutant secondary GBM (17). $0^{6}$-methylguanine-DNA methyltransferase (MGMT) promoter status is also used to stratify GBMs. Patients with methylated (or partially methylated) MGMT promoters are more likely to respond to the standard of care chemotherapeutic temozolomide, in contrast to those with unmethylated MGMT who are unlikely to benefit from the chemotherapeutic (18). In addition to temozolomide, the current standard of care includes surgical resection when possible and adjuvant radiation therapy, however, these treatments are associated with a median survival of only 15 months and a fiveyear survival rate of $6.8 \%(16,19,20)$.

Here we use CRISPR-based viability studies and CDNA rescue experiments to validate the paralog synthetic lethality between VRK1 and VRK2. Specifically, we show that in tumor cell lines with high VRK2 promoter methylation, and thus low VRK2 expression, knockdown of VRK1 induces cell death via G2/M arrest and DNA damage. We demonstrate that the kinase function of VRK1 is required for the synthetic lethality 
which posits that VRK1-targeting can be used as an approach to treat VRK2-methylated GBM.

\section{METHODS}

\section{Cell Culture}

Original cell lines were acquired from ATCC, ECACC, and JCRB. HAP1 isogenic cell lines were purchased from Horizon Discovery. All cell line stocks were routinely tested for mycoplasma. HAP1, LN229, YKG1, KNS60, U118MG, H4, LN18, T98G, YH13, KS1, KALS1, and SW1088 were maintained in Dulbecco's modified Eagle's medium (Gibco) supplemented with $10 \%$ fetal bovine serum (FBS; GeminiBio) and $1 \%$ sodium pyruvate (Gibco). U251MG cell lines were maintained in Eagle's minimal essential medium (EMEM; Quality Biological Inc) supplemented with $10 \%$ FBS. All cell lines were maintained in a cell culture incubator at $37{ }^{\circ} \mathrm{C}, 95 \%$ humidified air, and $5 \% \mathrm{CO}_{2}$ atmosphere. For detailed information see supplemental methods.

\section{DNA Constructs and Cell Line Engineering}

We used a dual vector lentiviral system for both CRISPR-Cas9 and tetracyclineinducible CRISPR-dCas9-KRAB cloning. All guide and cDNA sequences are reported in Supplemental Table 3. Lentivirus was generated by transiently transfecting Lenti-X 293T cells (Takara Bio) with lentiviral packaging mix (Cellecta), lipofectamine 3000 transfection reagent (ThermoFisher), and constructs diluted in Opti-MEM (Gibco). Virus was collected from the supernatant 48 hours post-transfection and filter sterilized using a $0.45 \mu \mathrm{m}$ filter. Cells were infected with lentivirus and polybrene and selected in 
medium containing puromycin, blasticidin, or geneticin antibiotic as determined by the construct. For detailed information see supplemental methods.

\section{Colony Forming Assays}

Cas9 expressing cells were seeded in tissue culture plates such that cells would reach $80-90 \%$ confluency in 14 days. The next day, cells were infected with lentivirus containing CRISPR guides and polybrene transfection reagent, and the following day, selected with $0.5 \mu \mathrm{g} / \mathrm{mL}$ puromycin. Cells were left to grow for 14 days and stained with crystal violet. For inducible CRISPR-dCas9-KRAB experiments, doxycycline was added to medium the day after seeding, and medium containing doxycycline was refreshed every 3-4 days during the 14-day growth period.

\section{Immunoblotting}

Cells were rinsed in cold PBS and lysed in RIPA buffer (CST) supplemented with protease and phosphatase inhibitors (ThermoFisher) and universal nuclease (ThermoFisher). Lysates were cleared of insoluble material by centrifuging at 20,000 $\mathrm{g}$ for 10 min at $4^{\circ} \mathrm{C}$ and protein concentration was determined with the BCA Protein Assay (ThermoFisher). For immunoblotting $20-40 \mu \mathrm{g}$ of protein in equal volumes were heated in LDS-sample buffer (Invitrogen) containing DTT for $5 \mathrm{~min}$ at $95^{\circ} \mathrm{C}$. Samples were centrifuged at $20,000 \mathrm{~g}$, separated by SDS-PAGE electrophoresis in $4-12 \%$ Bis-Tris gels (Invitrogen), and transferred to nitrocellulose membranes (Invitrogen). For detailed information see supplemental methods.

\section{Custom Antibody Generation}


Phosphorylated BAF polyclonal antibody was raised in rabbits against the peptide N-MTTpSQKHRDFVAEPM by ProSci, Inc. (Poway, CA). Antibodies that recognized phosphorylated BAF was separated from total antibodies by a two-step immunoaffinity purification. Serum was first loaded on a column with immobilized non-phosphopeptide, flow through was then loaded onto a column with immobilized phosphopeptide, and finally purified antibody was eluted.

\section{Murine Xenograft Studies}

The protocol was approved by the Institutional Animal Care and Use Committee (IACUC) of Pharmaron (Beijing, China) following the guidance of the Association for Assessment and Accreditation of Laboratory Animal Care (AAALAC). U251MG VRK2 low and U251MG VRK2 high cell lines were inoculated subcutaneously into 6- to 8week-old female NOG mice (10 million cells / animal with $50 \%$ high density matrigel in EMEM) and allowed to form palpable tumors. Once the tumors reached $\sim 150 \mathrm{~mm}^{3}$, the mice were assigned to treatment groups with similar mean tumor volumes and treated with either saline or doxycycline (25 mg/kg) QD by oral gavage. Tumors were measured twice weekly throughout treatment.

\section{Cell Cycle Analysis}

Cells were treated with doxycycline at the indicated concentrations and times, trypsinized, washed in PBS, fixed in $70 \%$ ethanol, and stained with Propidium lodide/RNase Staining Buffer (BD Biosciences). Individual cells were characterized for forward and side scatter and DNA content was determined in 10,000 cells as measured by flow cytometry (FACS; excitation at $488 \mathrm{~nm}$, emission measured using $600 \mathrm{~nm}$ 
bandpass filter) with an Attune cytometer (Thermo Fisher) or Novocyte cytometer (Agilent). Histograms and cell counts were generated using FlowJo X software.

\section{High-content immunofluorescence imaging}

Cells were cultured in CellCarrier-96 Ultra microplates (Perkin Elmer) in the presence or absence of $1 \mu \mathrm{g} / \mathrm{mL}$ doxycycline for 5 days. Cells were fixed, blocked, and incubated with primary and secondary antibodies as indicated. Plates were imaged using Harmony high-content imaging and analysis software (Perkin Elmer). Briefly, nuclei were identified using the "find nuclei" function, and nuclei located at the periphery were removed using the "remove border objects" feature. Nuclear envelope roundness was quantified using Alexa-488 signal, and based on roundness, abnormal nuclear envelope positive cells were scored by the software. For detailed information, see supplemental methods.

\section{Phospho- and Total Proteomics Analysis}

Cells were treated with and without doxycycline for the indicated times, washed twice in ice-cold PBS prior to harvesting. Proteins were digested using LysC/Trypsin and labeled for multiplexing using TMT labelling (ThermoFisher). Phospho proteins were collected using pSTY enrichment and fractionation and flow through from the PSTY enrichment was used for total protein-level analysis. All mass spectra were acquired on an Orbitrap Fusion Lumos coupled to an EASY nanoLC-1200 (ThermoFisher) liquid chromatography system. Heatmaps were generated on normalized scaled signal data using Morpheus Software (Broad Institute). Differential analysis between treatment groups was performed using an empirical Bayes method as in (21) and gene set 
enrichment analysis was calculated on proteins that were altered greater than +/- 2 Log fold-change. For detailed information, see supplemental methods.

\section{RESULTS}

\section{VRK1 is a synthetic lethal target in VRK2-methylated cancer cell lines}

To discover novel synthetic lethal interactions, we analyzed the large-scale cancer dependency database Achilles, where 808 cancer cell lines were screened with a genome-wide CRISPR Cas9 library to uncover genes essential for cell proliferation (22-24). We identified a subset of cell lines with low VRK2 expression that were sensitive to VRK1 knockdown (Fig 1A). Low VRK2 expression was primarily found in brain cancer and neuroblastoma cell lines, suggesting a neural lineage expression pattern. Genotype-Tissue Expression (GTEx) data reveals that normal human brain tissue has lower expression of VRK2 transcripts than other tissues, further suggesting a lineage-specific context (Fig S1A). Further analysis of CCLE data demonstrated that decreased expression of VRK2 strongly associated with increased promoter methylation (Fig 1B). VRK2 is rarely deleted or mutated in cancer cell lines or human tumors (Fig S1B-C) suggesting that the VRK2 methylation is the most likely cause of low VRK2 expression. To determine if VRK2-methylation occurs in primary human tumors, we analyzed data from The Cancer Genome Atlas (TCGA) low-grade glioma (LGG) and GBM datasets $(25,26)$. Approximately two-thirds of LGG and GBM have low VRK2 expression that correlates with VRK2 promoter methylation, demonstrating that the "VRK2-low" genetic context is present in human brain cancer (Fig 1C). As noted above, current prognostic markers for LGG and GBM are MGMT promoter methylation status and IDH1/2 mutation status, so we queried whether VRK2-low status co-occurs with 
either of these markers. About 32\% of LGG tumors with low VRK2 expression harbored mutations in IDH1 or IDH2, and approximately $55 \%$ of LGG and $21 \%$ of GBM tumors with low VRK2 expression had MGMT promoter methylation (Fig 1D, S1D).

\section{VRK1-VRK2 synthetic lethality in vitro and in vivo in GBM cell lines}

To validate the synthetic lethal relationship between VRK1 and VRK2, we obtained a VRK2-wildtype and -null isogenic cell line pair derived from the HAP1 model. We generated Cas9 derivatives of these cell lines and knocked out VRK1 using three different sgRNAs. Knockout of VRK1 was lethal in the VRK2-null cell line in a 14-day colony forming assay, whereas the HAP1 VRK2 wildtype cells continued to proliferate (Fig 2A). To control for Cas9 efficiency, we used sgRNA to the pan-lethal gene PLK1 and observed cell death in both cell lines. Immunoblots for VRK1 and VRK2 demonstrated 60-80 \% knockdown with all three VRK1 guides and confirmed VRK2 expression levels in the cell lines (Fig 2B). On-target knockdown was confirmed by rescuing the lethal phenotype in the HAP1 VRK2-null cells by expressing a CRISPR edit-resistant VRK1 cDNA (Fig 2C). To determine if the VRK1 kinase activity is important for the synthetic lethal interaction, we engineered VRK1-kinase dead and reduced activity mutations. Lysine 71 is the catalytic lysine in the VRK1 active site and the $\mathrm{K} 71 \mathrm{M}$ mutation eliminated VRK1 kinase activity as determined by in vitro phosphorylation activity on a VRK1 substrate (Fig S2A). Edit-resistant VRK1 kinase dead (K71M) mutant and a previously published reduced activity mutant (K179E) (27) failed to rescue the anti-proliferative phenotype, indicating that the kinase activity of VRK1 is required for the synthetic lethal interaction. Re-expression of VRK2 in the VRK2-null cells also rescued the lethality confirming that VRK2 loss in the VRK2-null 
cell line is the cause of the anti-proliferative phenotype. Immunoblots for VRK1 and VRK2 confirm the expression of the respective cDNA constructs (Fig 2D). To further evaluate the synthetic lethal interaction in VRK2-methylated GBM cell lines, we tested the effects of VRK1 knockdown in a panel of VRK2-methylated and -unmethylated GBM cell lines (Fig S2B). VRK2-methylated cell lines were more sensitive to VRK1 knockdown than VRK2-unmethylated in a 14-day colony forming assay (Fig 2E-F). The data were quantified by normalizing colony intensity compared to intron cutting controls and were controlled for Cas9 efficiency by subtracting the colony intensity of PLK1 panlethal controls (Fig 2G). We further tested sensitivity to VRK1 CRISPR knockout in two non-GBM lines, RKO and SNU-398 and observed no proliferative defect in a similar colony forming assay (Fig S2C).

To extend our in vitro findings to the in vivo setting, we first established doxycycline-inducible VRK1 knockdown in the VRK2-methylated U251MG GBM model. Clonal U251MG cells expressing ubiquitous dCas9-KRAB and doxycycline-inducible CRISPR guides for the VRK1 promoter (henceforth referred to as "VRK2-low") were sensitive to knockdown of VRK1 in a 14-day colony forming assay (Fig 3A). We generated "VRK2-high" derivatives of the clonal cell lines by re-expressing ubiquitous VRK2 cDNA and observed a full rescue of the anti-proliferative phenotype (Fig 3B). Protein levels of VRK1 and VRK2 were verified by immunoblotting (Fig 3C). Similarly, dox-inducible knockdown of VRK1 in a VRK2-unmethylated LN229 GBM cell line showed no proliferative defect in vitro (Fig S3A-B). To evaluate the synthetic lethal interaction in vivo, mice harboring established, subcutaneous xenografts of U251MG VRK2-low and VRK2-high derivative cell lines were treated with doxycycline or saline 
solution via oral gavage. A subset of tumors from all arms of the study were collected at seven days post-treatment and immunoblots demonstrated successful dox-induced VRK1 knockdown in both models and sustained VRK2 expression in the VRK2-high tumors (Fig 3D). Tumors in the VRK2-low derivative models regressed after 36 days on treatment compared to the vehicle-treated arm. Doxycycline treatment was stopped in five mice and continued in four mice at day 50 at which time the tumors started to regrow in both arms (Fig 3E). Immunoblots of these tumors at endpoint (day 60) reveal re-expression of VRK1 protein suggesting selection pressure to maintain VRK1 expression is required for survival in vivo in the absence of VRK2 expression (Fig 3G). Tumors in the VRK2-high group continued to grow in the presence of doxycycline compared to the saline arm (Fig 3F), suggesting that VRK2 is a key predictor of response to VRK1 inhibition in this model.

\section{VRK1 regulates BAF activity to maintain nuclear envelope integrity}

Since VRK1 plays an important role in cell cycle progression (11), we queried whether VRK1 knockdown led to aberrant cell cycling in the VRK2-low context. Doxycycline-induced VRK1 knockdown for seven days provoked a G2/M arrest in the U251MG VRK2-low cell line as demonstrated by flow cytometry of propidium iodidestained cells (Fig 4A, S4A). The VRK2-high U251MG derivative continued to cycle, suggesting that the mechanism of VRK1-VRK2 synthetic lethality involved a G2/M arrest. A similar G2/M arrest was also observed in the HAP1 VRK2-null, but not HAP1 VRK2-wildtype cells upon VRK1 knockdown (Fig 4B, S4B). Since VRK1 phosphorylates several substrates involved in mitosis, we profiled the phosphorylation status of the reported substrates histone $\mathrm{H} 3$ (T3, S10), p53 (T18) and BAF (S4). We were unable to 
detect any phosphorylation of p53 at Thr18 with several commercially available antibodies (data not shown). We did not observe significant alterations in the phosphorylation of Histone $\mathrm{H} 3$ at Thr3 and Ser10 (Fig S4C). As there is no commercially available antibody for the detection of phosphorylated BAF, we generated a custom polyclonal phospho-BAF (S4) antibody. Lambda phosphatase-treatment of HAP1 lysates demonstrates that this antibody recognizes a mixture of phospho-BAF and total-BAF proteins (Fig 4SD). Phosphorylation of BAF (S4) was depleted upon VRK1 knockdown and restored upon VRK2 re-expression (Fig 4C, S4C). A similar alteration in phosphorylation of BAF (S4) was observed in the HAP1 VRK2-null cell line with VRK1 knock-out (Fig 4D). Consistent with previously published data, total BAF was also depleted upon VRK1 knockdown in both cell line models $(28,29)$. BAF, encoded by the BANF1 gene, is a highly conserved chromatin binding factor that regulates postmitotic nuclear envelope formation by linking nuclear envelope proteins to the DNA (30). Depletion of BAF results in aberrant nuclear envelope formation, nuclear blebbing, and multinucleation (31). Knockdown of VRK1 for five days led to abnormal nuclear envelope, nuclear lobulation, multinucleation and arrested mitotic spindles as determined by Lamin B immunofluorescent staining (Fig 4E). This phenotype was not due to a doxycycline effect since a non-targeting control guide did not produce nuclear envelope defects in the presence of doxycycline (Fig S4D). Re-expression of VRK2 rescued the abnormal nuclear envelope phenotype (Fig 4E-F) suggesting that the synthetic lethality depends, in part, on the VRK1 and VRK2 substrate BAF.

The VRK1-VRK2 synthetic lethality activates the DNA damage response pathway 
To further understand the mechanism underlying the synthetic lethality, we performed global phospho- and total-proteomic profiling in the U251MG VRK2-low and VRK2-high cell lines at five and seven days post-doxycycline treatment. Global changes in the proteome and phospho-proteome were more marked at seven days than five days, and thus, we performed differential analysis between VRK2-low and VRK2-high cell lines in the presence of doxycycline at the seven day timepoint in both datasets (Fig 5A, Supplemental Table 1). Consistent with our previous findings, differential analysis of proteomic profiling revealed notable GSEA enrichment of proteins involved in G2/M arrest such as PLK1, AURKA, AURKB, BIRC5, CDC6 and CCNB1 at seven days post doxycycline treatment (Fig 5B-C, Supplemental Table 2). The changes in G2/M proteins were observed as early as five days (Fig S5) and at the later seven day timepoint, we also observed an accumulation of proteins involved in DNA repair such as RAD51, PCNA, RPA3 and members of the RFC complex. The phospho-proteomics data also indicate a G2/M arrest due increased phosphorylation of CDK1 (CDC2) at Tyr15 which inhibits the progression of the cell cycle. We additionally observed increased phosphorylation of CDC20 at S41 and T70 which is reported to accumulate during mitosis (32). Consistent with the nuclear envelope defect in VRK2-low cells upon VRK1 knockdown, we observed an accumulation of phosphorylated Lamin A/C and TMPO which signal nuclear envelope breakdown (33). Phosphorylated BAF was not detected in this dataset likely due to the small molecular weight and low abundance of the protein. The G2/M and DNA damage response proteins were not altered in the VRK2high cell line suggesting that these processes are involved in the VRK1-VRK2 synthetic lethal interaction. A subset of G2/M and DNA damage response markers were validated 
by immunoblotting (Fig $5 \mathrm{E}$ ). These data demonstrate that the synthetic lethal mechanism of VRK1 perturbation in a VRK2-low GBM cell line is a G2/M cell cycle arrest and subsequent DNA damage.

\section{Patient-derived GBM models maintain the VRK2-low state}

Since available cell line models of GBM are not representative of the heterogeneous nature of the disease (34), we evaluated VRK2 expression levels was present in patient-derived GBM models. We performed immunoblotting for VRK2 in a panel of patient-derived GBM models and observed low VRK2-expression in seven out of nine models (Fig 6A). Measurement of VRK2 mRNA transcript levels by quantitative RT-PCR corroborated the immunoblot results (Fig S6). Since GBM contains selfrenewing, tumorigenic cancer stem cells (CSCs) that contribute to tumor initiation and therapeutic resistance (35), we asked whether VRK2 expression differs between differentiating and non-differentiating media conditions. Each of the models were cultured either in CSC- (non-adherent, serum-free) or in non-CSC- (adherent, with serum) promoting conditions as previously described (36). VRK2 protein levels measured by immunoblotting remained unchanged in both media conditions suggesting stability of the promoter methylation regardless of differentiation status (Fig 6B). These data demonstrate that the VRK2-low context is maintained in patient-relevant GBM models.

\section{DISCUSSION}


Recent data from genome-scale CRISPR-Cas9 screening across hundreds of cancer cell lines have resulted in nomination of multiple novel targets for potential therapeutic development (37-40). In this study, we identified VRK1 as a paralog synthetic lethal target in VRK2-methylated GBM and neuroblastoma cell lines. Using CRISPR-based genetic tools, we demonstrate that knockdown of VRK1 in VRK2-null and VRK2-low expressing GBM cell lines is lethal, and results in defective nuclear envelope formation, G2/M arrest, and subsequent DNA damage. The synthetic lethal interaction is recapitulated in vivo in a VRK2-methylated U251MG GBM xenograft model, where VRK1 knockdown leads to tumor regressions. Xenografts from an isogenic VRK2-high U251MG cell line are insensitive to VRK1 knockdown, suggesting that the sensitivity depends solely on VRK2 expression. Importantly, our study demonstrates that the enzymatic activity of VRK1 is required for the VRK1-VRK2 synthetic lethality, which provides a path for small molecule drug discovery. Patient data indicates that the VRK2-methylated/VRK2-low context is present and may be common in LGG and GBM tumors. Taken together, these findings suggest that VRK1 is a promising synthetic lethal drug target in VRK2-methylated brain tumors, an aggressive indication with few therapeutic strategies currently available.

Past studies have identified synthetic lethal relationships among paralog genes such as SMARCA2-SMARCA4, ARID1A-ARID1B, and CREBBP-EP300 (41-43). The advantage of a synthetic-lethal therapeutic approach for cancer is the inherent large therapeutic index which maximizes anti-tumor efficacy while minimizing dose-limiting on-target toxicities (1). However, creating a paralog-selective inhibitor with these qualities hinges on developing a highly selective inhibitor that spares the non-target 
paralog despite nearly identical protein sequence homologies. Through mutant and wildtype cDNA rescue experiments, we show that the kinase function of VRK1 is essential in the synthetic lethal interaction. We believe that selectively targeting VRK1 over VRK2 may be possible as the kinase domains of VRK1 and VRK2 have approximately $80 \%$ protein sequence identity. Recent structural biology analysis revealed differential mechanisms for stabilization of an ATP-competitive inhibitor in the binding pocket of VRK1 compared to VRK2 $(44,45)$, further supporting the possibility for development of a paralog-selective VRK1 kinase inhibitor.

VRK1 is one of the most abundant nuclear kinases in human cells and its overexpression is associated with poor prognosis in many solid tumors including GBM and neuroblastoma (46-49). We have shown for the first time that tumors with the genetic context of VRK2-methylation may benefit from selective inhibition or degradation of VRK1. Publicly-accessible cancer cell line data suggest that the VRK2methylated context is restricted to cancer stemming from the neural lineage, i.e., GBM and neuroblastoma. Healthy human tissue expression data from the GTEx project further corroborates the lineage effect with lower VRK2 expression in neural-derived tissues compared to other tissues (Figure S1A). Additional evidence of a lineage effect comes from genetics on people with germline mutation of $V R K 1$, which results in a neurological disease that manifests as prenatal microcephaly with pontocerebellar hypoplasia (50-53). Paralleling the human disease, mice with partial Vrk1 knockdown have reduced brain weight and mild motor dysfunction (54). We posit the susceptibility of the developing brain to loss of VRK1 may be due to a naturally occurring synthetic 
lethal interaction stemming from reduced VRK2 expression in the neural cells compared to other lineages.

Although many substrates have been reported for VRK1 (55), our results demonstrate that BAF may be critical in the VRK1-VRK2 synthetic lethal interaction. We observe that knockdown of VRK1 leads to downregulation of BAF activity and results in a nuclear envelope defect that phenocopies BAF depletion $(31,56-59)$. It is interesting to note that we and others observe decreases in both phosphorylated and total BAF, which may be due to protein instability in the absence of phosphorylation, a hypothesis that will require further testing $(28,29)$. In addition to post-mitotic nuclear envelope assembly, BAF is involved in regulation of the DNA damage response and intrinsic immunity (60). Inherited germline BANF1 mutations, which results in instability of the BAF protein, cause Nestor-Guillermo Progeria Syndrome, a premature aging disease characterized by genome instability and accumulation of DNA damage $(61,62)$. Based on this study and previously published data, we postulate that the increased DNA damage response observed is due to genomic instability arising from the defective postmitotic nuclear envelope. Interestingly, BAF also regulates cell-intrinsic immunity during mitosis by preventing cytosolic cGAS activation on genomic self-DNA (63). BAF dynamically outcompetes cGAS for DNA binding and prohibits the formation of DNAcGAS complexes which are essential for inducing the type I interferon response. We did not observe cGAS activation or type I interferon response in our proteomics dataset suggesting that the pathway is not involved in the VRK1-VRK2 synthetic lethality. Taken together our study suggests that BAF plays a role in the VRK1-VRK2 synthetic lethal interaction. In VRK2-intact cells, VRK1 knockdown does not affect BAF activity due to 
compensation by VRK2, and results in formation of a smooth nuclear envelope at the end of mitosis. In VRK2-low cells, VRK1 knockdown leads to depletion of phosphorylated and total BAF, resulting in aberrant nuclear envelope with blebbing, lobulation and micronucleation. The cells arrest at the end of mitosis, accumulate DNA damage, and eventually die (Fig 6C).

Despite aggressive treatments for newly diagnosed GBM, almost all patients relapse with more aggressive tumors with minimal treatment options within one to two years. To date, efforts to develop treatments based on genetic alterations such as EGFR amplification, CDKN2A loss, TERT promoter mutation or PTEN mutation have been unsuccessful (64). Our findings indicate that VRK1 is a potential target for synthetic-lethal therapy in VRK2-low GBM, an aggressive indication with few therapeutic options. Interestingly, a recent study demonstrated that knockdown of VRK1 in vitro synergizes with temozolomide treatment by augmenting the DNA damage response (65). Together these data suggest that a VRK1 inhibitor may be used as a single agent or in combination with the current standard of care to augment therapeutic response.

The results of this study uncover a novel paralog synthetic-lethal interaction between VRK1 and VRK2 in GBM. We demonstrate that knockdown of VRK1 is lethal in VRK2-methylated GBM cell lines in vitro and in vivo, and the kinase activity of VRK1 is important in the interaction. The mechanism underpinning the lethality is BAF deregulation resulting in aberrant nuclear envelope formation, G2/M arrest, and subsequent DNA damage. These findings support the significant therapeutic potential of a VRK1 inhibitor in VRK2-methylated GBM. 


\section{DATA AVAILABILITY}

Mass spectrometry raw files will be uploaded to the UCSD MassIVE repository when accepted for publication.

\section{AUTHOR CONTRIBUTIONS}

Conceptualization, F.L, A.H, and N.E.; Methodology, J.A.S., S.R.M, J.L.E., M.B., W.Z., M.Z., J.D.L., E.W., F.L., and N.E.; Formal Analysis, J.A.S., S.R.M, J.L.E., M.B., E.E.MH., K.M., W.Z., F.L. and N.E.; Data Acquisition, J.A.S., J.L.E., M.B., E.M-H., K.M., W.Z.,

S.Z., F.L. and N.E.; Writing - Original Draft, J.A.S and N.E.; Writing - Review and Editing, N.E., J.L., J.N.A, and Y.Cs.; Visualization, N.E.; Supervision, J.L., E.W., J.N.A, Y.C., F.L, A.H., and N.E.

\section{ACKNOWLEDGEMENTS}

We thank Dr. Douglas Whittington for structural analysis of VRK1 and Dr. Maria "Masha" Alimova for assistance with high-content imaging analysis. 


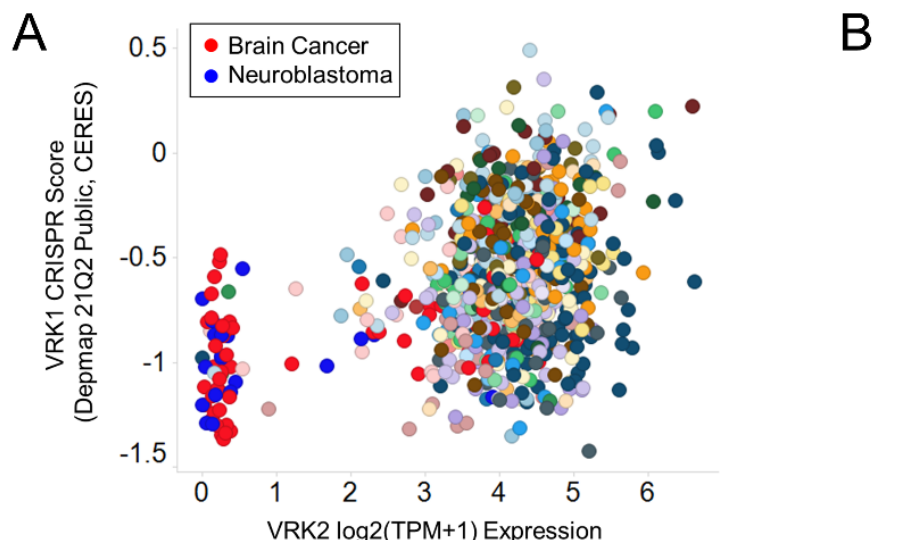

$$
\text { (21Q2 Public) }
$$

$\begin{array}{lll}\text { Breast Cancer } & \text { Bile Duct Cancer } & \text { Fibroblast } \\ \text { Colon/Colorectal Cancer } & \text { Bladder Cancer } & \text { Gallbladder Cancer } \\ \text { Endometrial/Uterine Cancer } & \text { Bone Cancer } & \text { Gastric Cancer } \\ \text { Esophageal Cancer } & \text { Brain Cancer } & \text { Head and Neck Cancer }\end{array}$

C

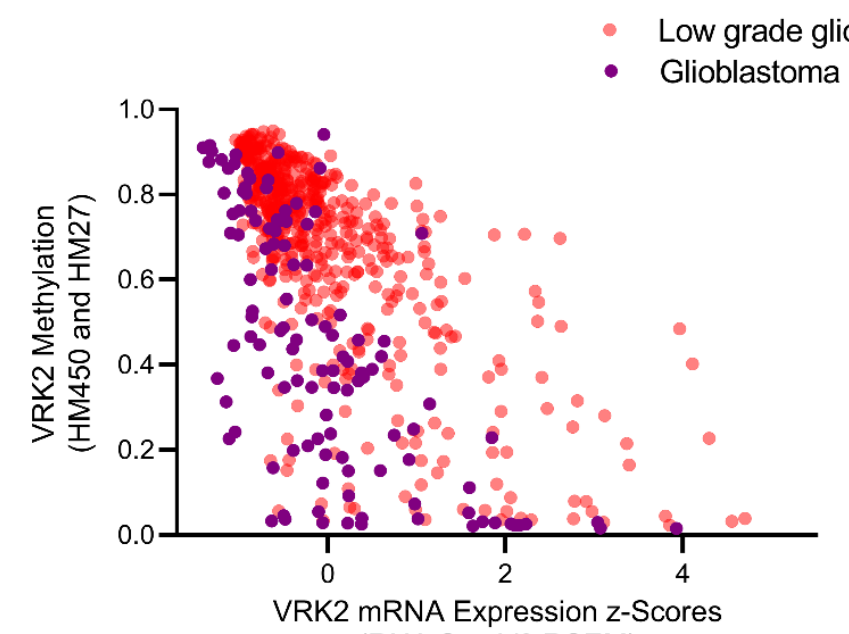

(RNA Seq V2 RSEM)

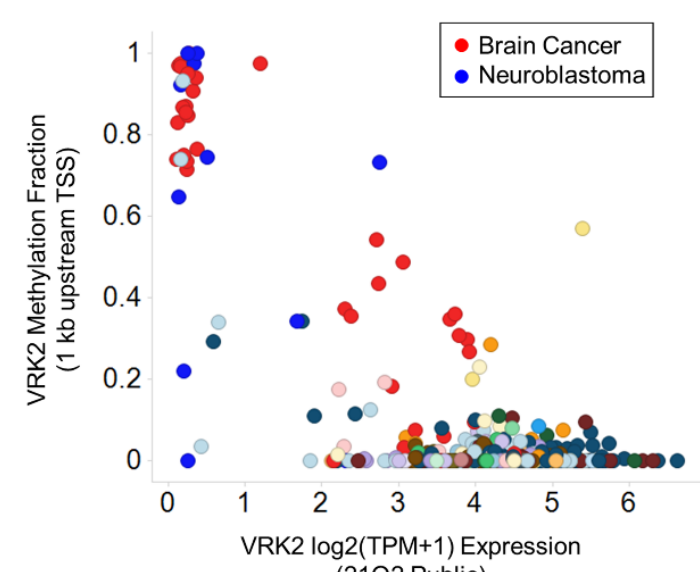

(21Q2 Public)

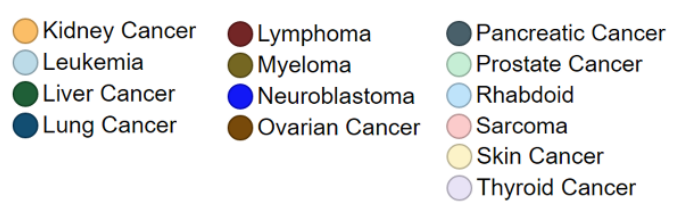

D

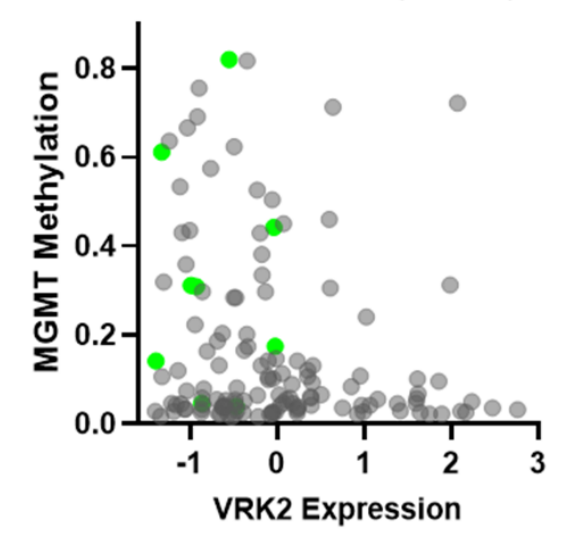

Low grade glioma (TGCA)

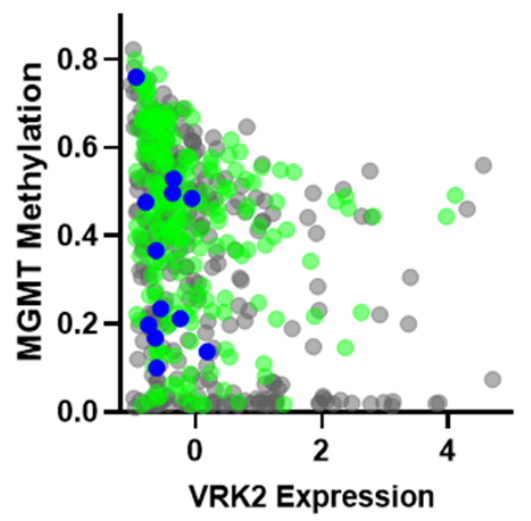

- No IDH mutation

- IDH1 mutation

- IDH2 mutation 
Figure 1. VRK2-methylated Glioblastoma and Neuroblastoma Cell Lines are Sensitive to VRK1 Loss.

(A) Scatter plot depicting VRK2 expression and VRK1 CRISPR knockdown sensitivity score in 783 cancer cell lines. Color coded by primary lineage.

(B) Scatter plot depicting VRK2 expression and VRK2 promoter methylation in 902 cancer cell lines. Color coded by primary lineage.

(C) Scatter plot depicting VRK2 Expression and VRK2 Methylation for 530 low grade glioma (LGG; peach) and 116 glioblastoma (GBM; purple) tumors

(D) Scatter plots with VRK2 expression and MGMT methylation for tumors as in (C), color coded by IDH mutation status. 

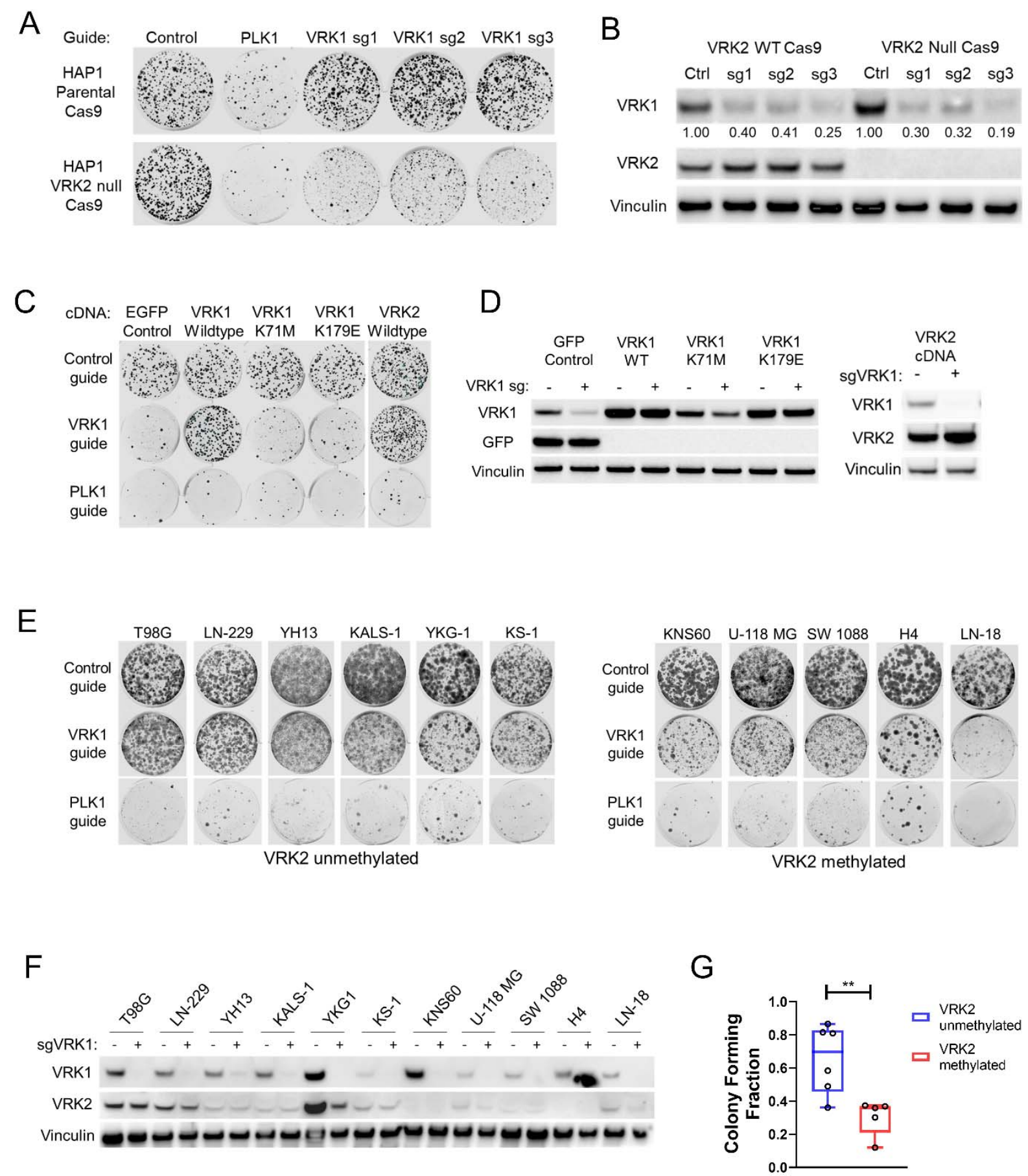

Figure 2. VRK1 is Synthetic Lethal in VRK2-null and VRK2 Methylated Cell Lines.

(A) 14-day colony forming assay in HAP1 parental and VRK2-null cells with CRISPR 
knockdown for intron-cutting negative controls, positive control PLK1 and three VRK1 guides.

(B) Immunoblots from (A) at 3 days. Quantification of VRK1 bands, normalized to Vinculin and relative to intron-cutting-controls, are indicated below the blot.

(C) 14-day colony forming assays of VRK1 knockdown in HAP1 VRK2-null cell line with ectopic expression of the indicated cDNA constructs.

(D) Immunoblots from (C) at 3 days.

(E) 14-day colony forming assays of VRK1 knockdown in a panel of VRK2unmethylated and -methylated GBM cell lines.

(F) Immunoblots from (E) at 3 days depicting VRK1 and VRK2 protein expression.

(G) Quantification of colony forming intensities of $(E)$ from two biological replicates, corrected for Cas 9 cutting efficiency, ${ }^{* *} \mathrm{p}<0.005$, two-tailed t-test 

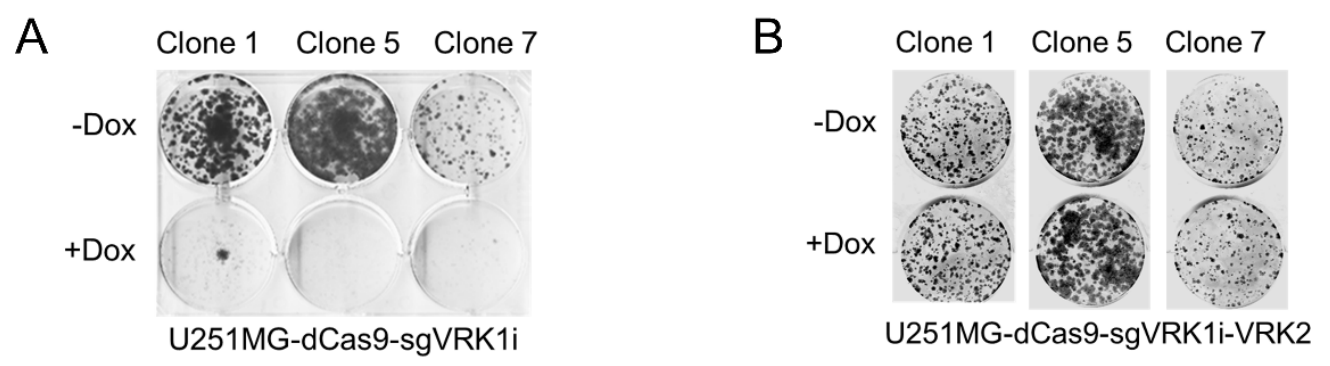

C

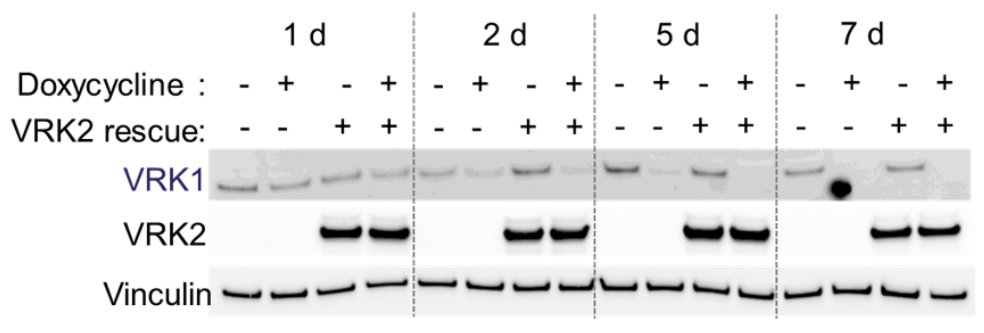

D

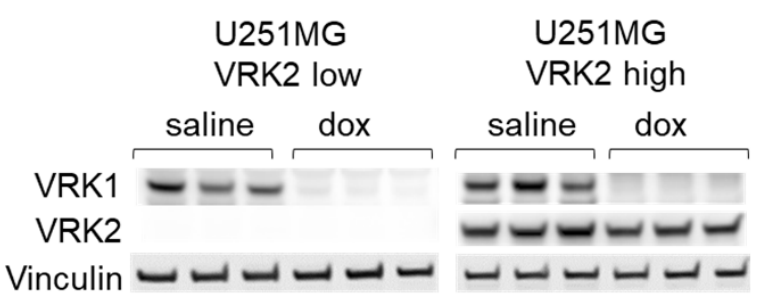

E

U251MG VRK2 low

F
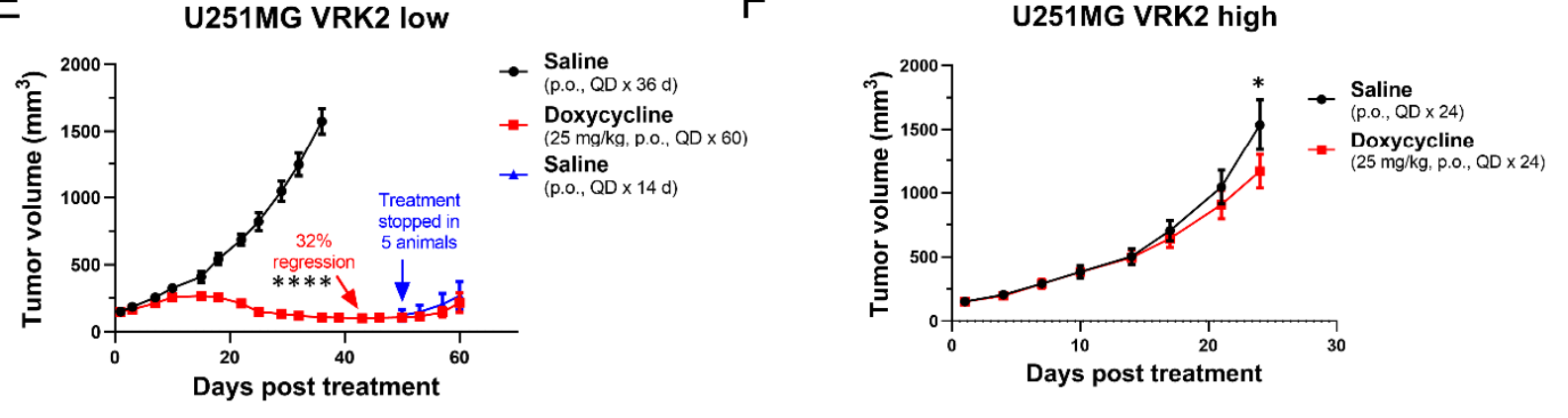

G

U251MG VRK2 low

$\begin{array}{lcccc}\begin{array}{c}7 d \\ \text { saline }\end{array} & \begin{array}{c}37 d \\ \text { saline }\end{array} & \begin{array}{c}7 d \\ \text { dox }\end{array} & \begin{array}{c}60 d \\ \text { dox }\end{array} & \begin{array}{c}46 d \text { dox }+ \\ 14 d \text { saline }\end{array} \\ \text { VRK1 } & \text { VRK2 } & & \end{array}$


Figure 3. The VRK1-VRK2 Synthetic Lethality is Maintained In Vivo.

(A) 14-day colony forming assays in U251MG VRK2-low cell line the absence or presence of $1 \mu \mathrm{g} / \mathrm{mL}$ doxycycline to induce VRK1 knockdown

(B) Assay similar to (A) in the U251MG VRK2-high cell line

(C) Immunoblots from (A) and (B) at 1-, 2-, 5- and 7-days post doxycycline treatment

(D) Immunoblots from U251MG VRK2-low and VRK2-high xenografts in tumor-bearing mice treated with or without $25 \mathrm{mg} / \mathrm{kg}$ doxycycline QD for 7 days.

(E) Tumor growth curves in mice bearing established $150 \mathrm{~mm}^{3}$ U251MG VRK2-low xenografts were treated with the indicated treatments. Data are presented as mean tumor volume \pm SEM with 9 mice/data point up to Day $42,{ }^{* * * *} \mathrm{p}<0.0001$ (two-tailed $\mathrm{t}$ test).

(F) Tumor growth curves as in (E) with U251MG VRK2-high xenografts. Data are presented as mean tumor volume \pm SEM with 9 mice/data point, ${ }^{*} \mathrm{p}=0.032$ (two-tailed $\mathrm{t}$ test).

(G) 7-day and endpoint immunoblots tumors from (E). 
A
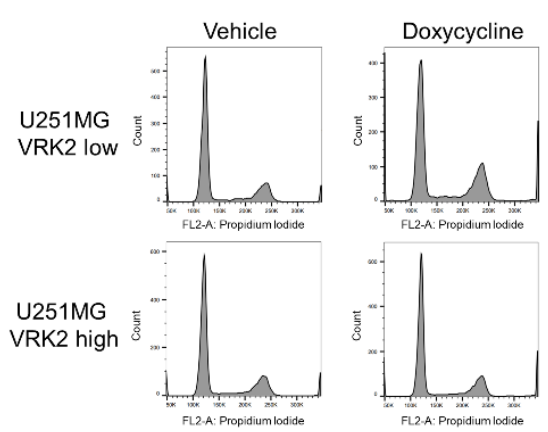

C

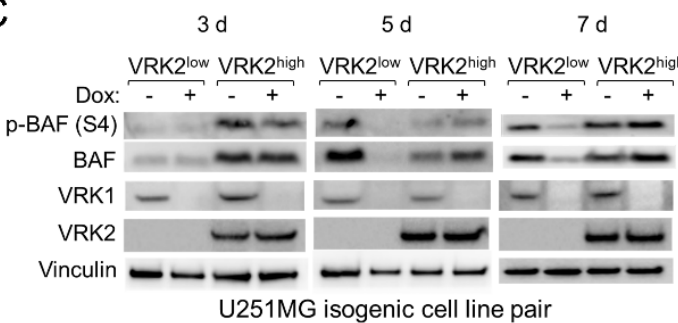

E

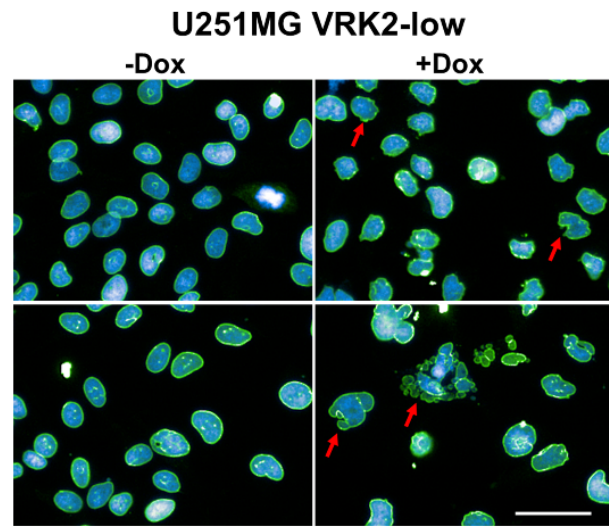

Lamin B/Hoechst

$\mathrm{F}$

Abnormal Nuclear Envelope

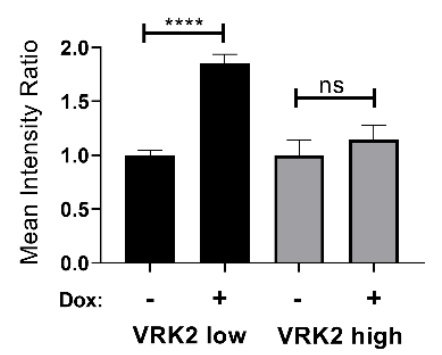

Figure 4. VRK1 Knockdown Results in G2/M Arrest, BAF Deregulation and
B
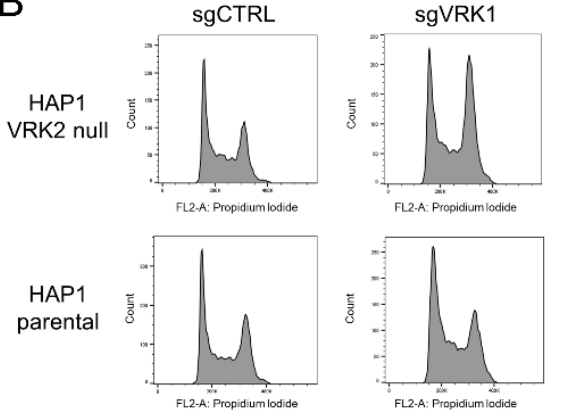

D

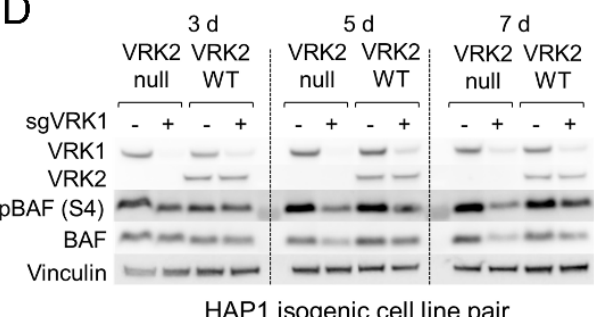

HAP1 isogenic cell line pair

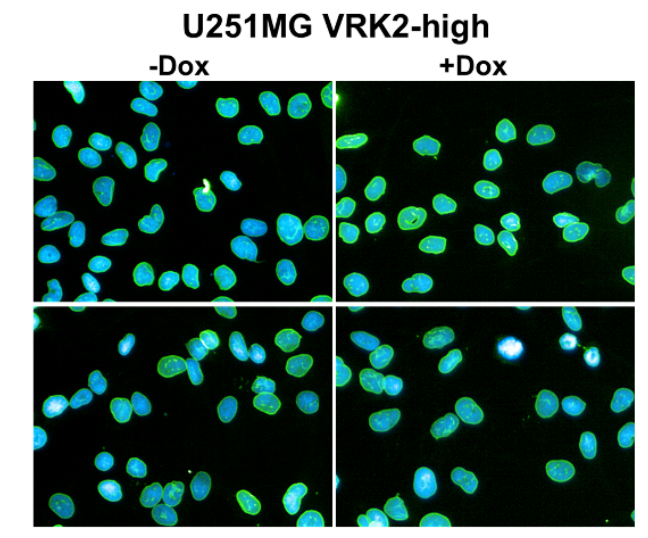

U251MG VRK2-high

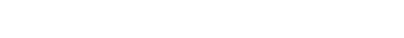


VRK2-low and VRK-high cells in the presence or absence of $1 \mu \mathrm{g} / \mathrm{mL}$ doxycycline for 7 days

(B) Cell cycle distributions were determined by flow cytometric analyses in HAP1 VRK2null and parental cells 5 days after VRK1 CRISPR knockdown (C) Immunoblots of U251MG VRK2-low and VRK2-high cells treated with or without doxycycline for 3-, 5- and 7-days.

(D) Immunoblots of HAP1 VRK2-null and parental cells knocked out with VRK1 or intron-cutting control guides for 3-, 5-, and 7-days.

(E) U251MG VRK2-low and VRK2-high cells were treated with or without doxycycline for 5 days and immunostained for Lamin B (green) and Hoechst (blue) and imaged by high-content imaging. Scale bar $50 \mu \mathrm{m}$.

(F) Quantification of abnormal nuclear envelope from (E). ${ }^{* * *} p<0.0001$, ns $=$ not significant (two-tailed t-test) 
A

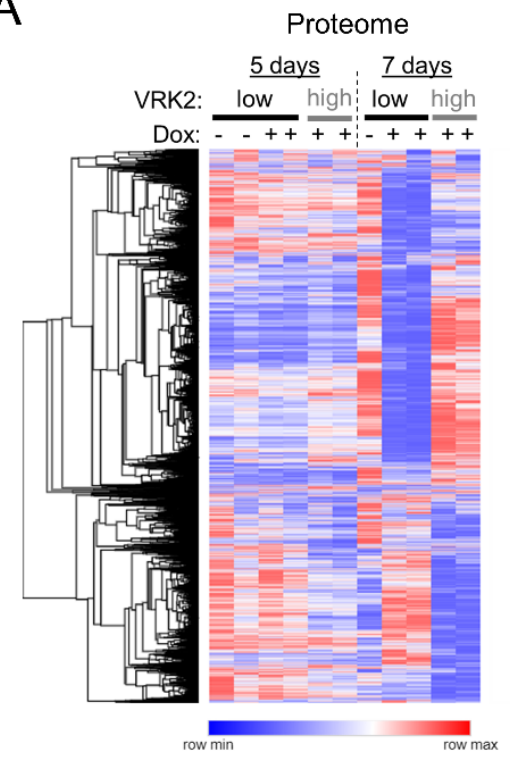

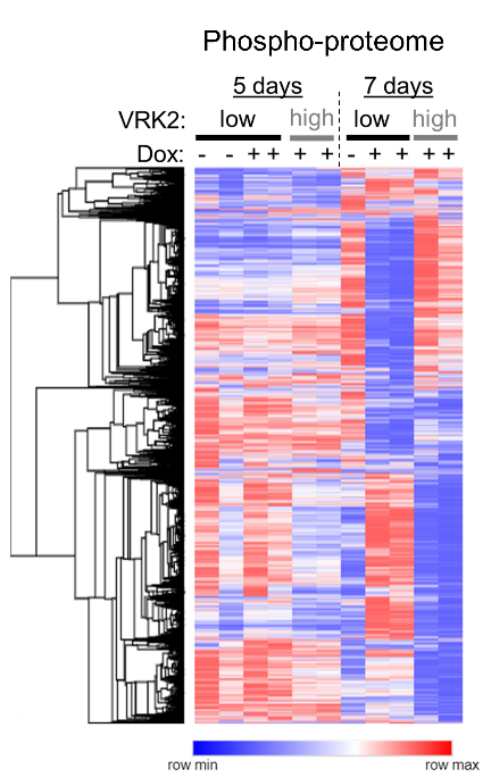

B
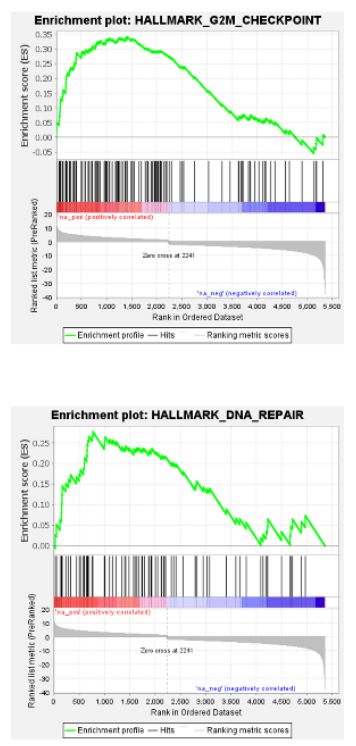

C

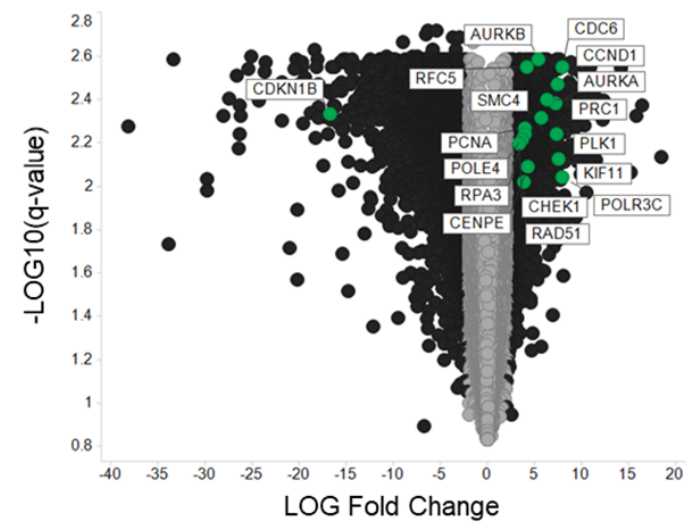

D $\quad 2.6$

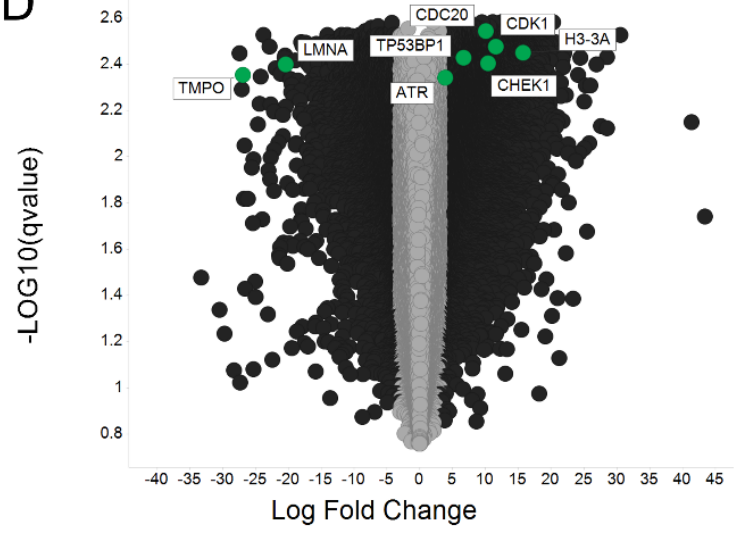

E

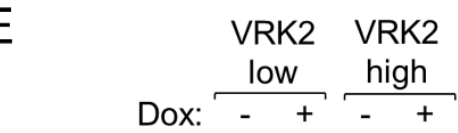

Cyclin D1 - - -

Cyclin A2

Cyclin B1

p-CDK1(Y15) - - -

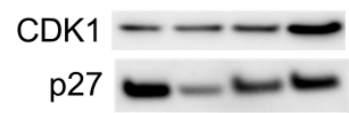

Cdc6

PLK1 - - -

AURKA - =

AURKB - - - -

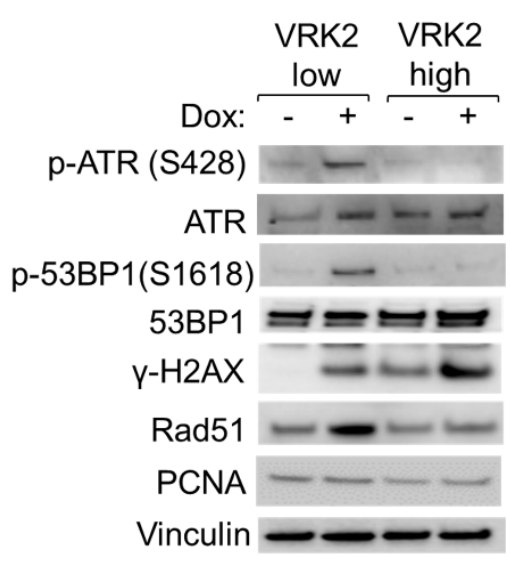


Figure 5. Phospho- and Total-Proteomics Reveals DNA Repair Pathways are Activated upon VRK1 Knockdown in VRK2-low Cells.

(A) Heat maps showing total-proteomics (left) and phospho-proteomics (right) in VRK2low and VRK2-high U251MG cells at 5- and 7-days post-doxycycline.

(B) Gene Set Enrichment Analysis (GSEA) of 7-day total proteomics data in the doxycycline conditions.

(C) Volcano plots differential expression analysis of total proteomic data. The x-axis represents $\log 2$ fold change and the $y$-axis represents the false discovery rate $[-\log 10(q-$ value)]. Black circles - proteins with greater than $+/-2$ log-fold change.

(D) Volcano plots differential expression analysis of phospho-proteomic data. The x-axis represents $\log 2$ fold change and the $y$-axis represents the false discovery rate $[-\log 10$ (qvalue)]. Black circles - proteins with greater than $+/-2$ log-fold change.

(E) Immunoblots of select proteins from U251MG VRK2-low and VRK2-high cells treated with or without doxycycline for 7 days. 


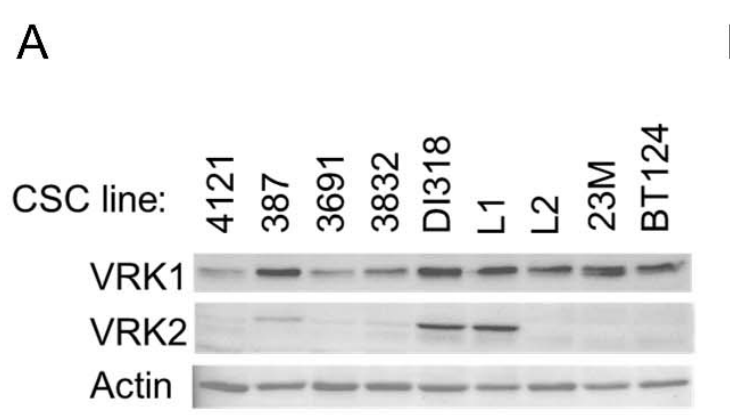

B
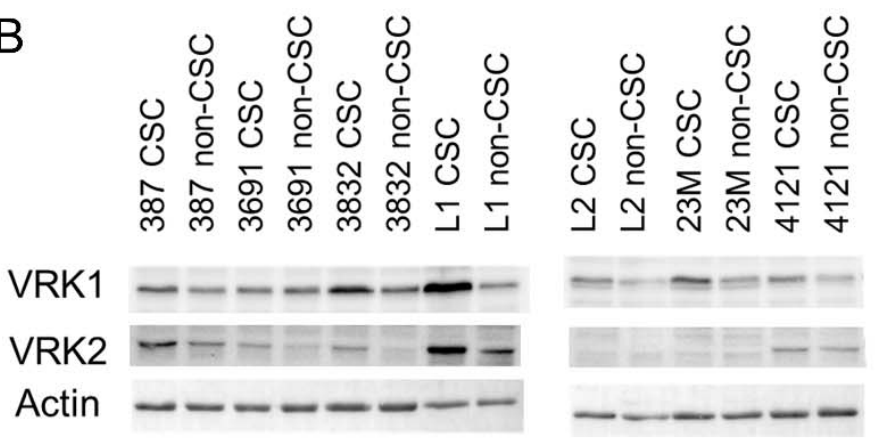

C

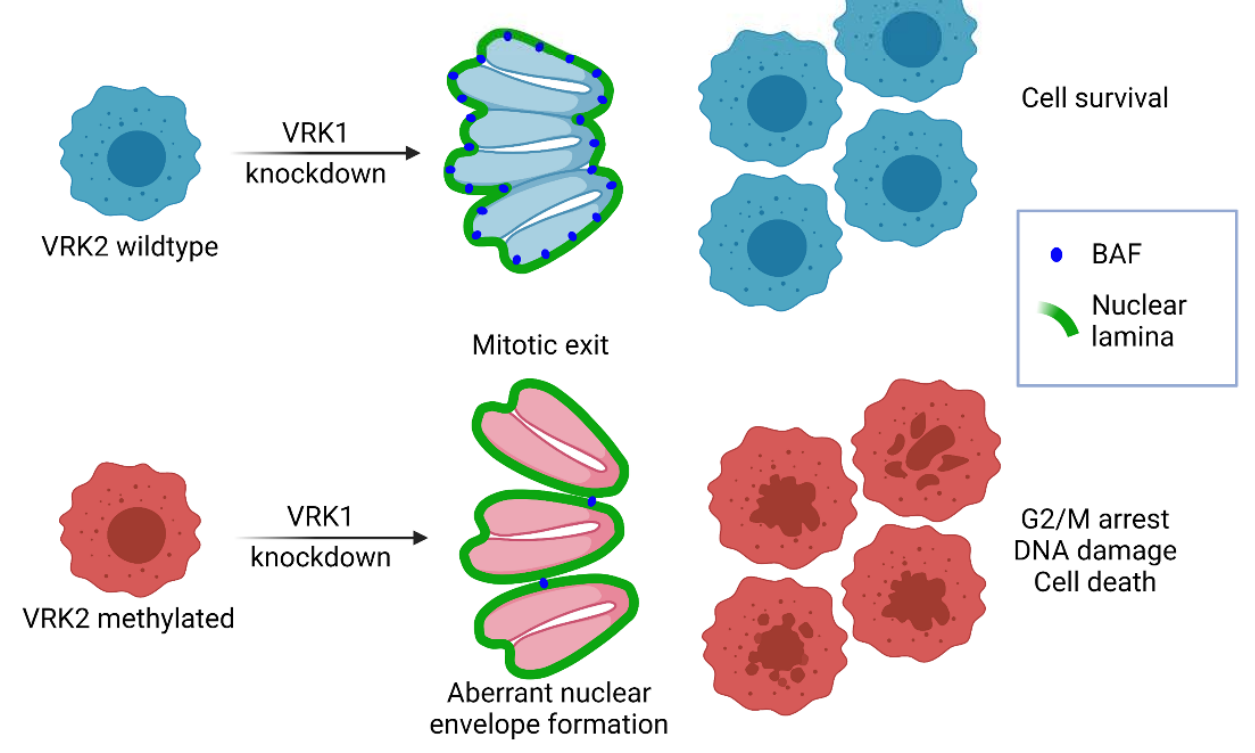

Figure 6. VRK2-methylated Context is Maintained in Patient-Relevant

\section{Glioblastoma Models}

(A) Immunoblots from 9 GBM CSC models

(B) Select CSC models were cultured in CSC or non-CSC medium and immunoblotted for VRK1 and VRK2 protein.

(C) Schematic depicting mechanism of the VRK1-VRK2 synthetic lethality. 
A

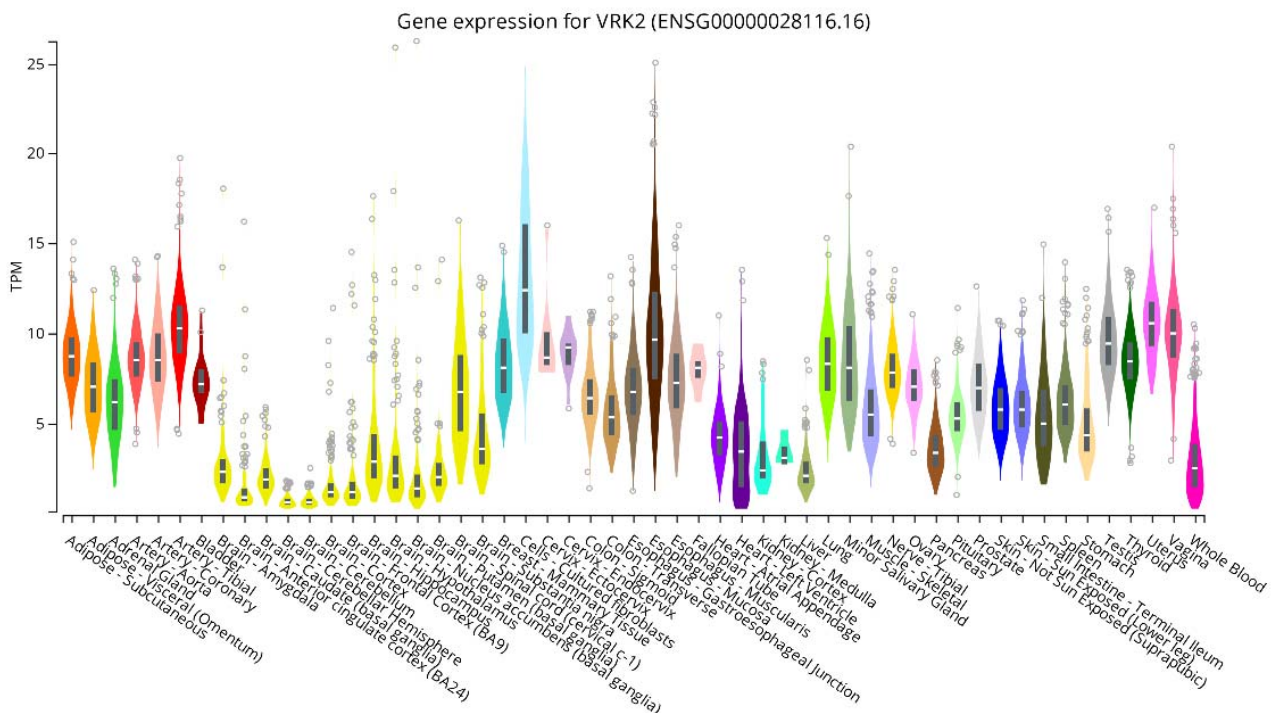

Data Source: GTEx Analysis Release V8 (dbGaP Accession phs000424.v8.p2)

B Cell line data (CCLE)

\begin{tabular}{cccc}
\hline Mutation type & Damaging & $\begin{array}{c}\text { Other non- } \\
\text { conserving }\end{array}$ & Deletion \\
\hline Percentage & $1.89 \%$ & $1.75 \%$ & $0.07 \%$ \\
\hline
\end{tabular}

C Patient tumor data (TCGA PanCancer)

\begin{tabular}{ccccc}
\hline Alteration type & $\begin{array}{c}\text { Truncating } \\
\text { mutation }\end{array}$ & $\begin{array}{c}\text { Splice } \\
\text { mutation }\end{array}$ & $\begin{array}{c}\text { Missense } \\
\text { mutation }\end{array}$ & Deletion \\
\hline Percentage & $0.15 \%$ & $0.03 \%$ & $0.65 \%$ & $0.10 \%$ \\
\hline
\end{tabular}

D MGMT methylation

\begin{tabular}{cccc}
\hline TCGA Cohort & $\begin{array}{c}\text { Number of } \\
\text { VRK2-low } \\
\text { cases * }\end{array}$ & Total cases & $\begin{array}{c}\text { Calculated } \\
\text { percentage }\end{array}$ \\
\hline LGG & 289 & 530 & $54.53 \%$ \\
\hline GBM & 29 & 138 & $21.01 \%$ \\
\hline *selection criteria: MGMT methylation $>0.2$ and VRK2 expression $<0$
\end{tabular}

IDH1/2 mutation

\begin{tabular}{cccc}
\hline TCGA Cohort & $\begin{array}{c}\text { Number of } \\
\text { VRK2-low } \\
\text { cases* }\end{array}$ & Total cases & $\begin{array}{c}\text { Calculated } \\
\text { percentage }\end{array}$ \\
\hline LGG & 168 & 530 & $31.70 \%$ \\
\hline *selection criteria: IDH1/IDH2 mutation and VRK2 expression <0
\end{tabular}

\section{Supplemental Figure 1.}

(A) VRK2 mRNA expression across all normal tissues (B) Percentage of VRK2 alterations in cancer cell lines (C) Percentage of VRK2 alterations in human tumors (D) Percentage of VRK2 low cases in MGMT-methylated and IDH mutant tumors 
A

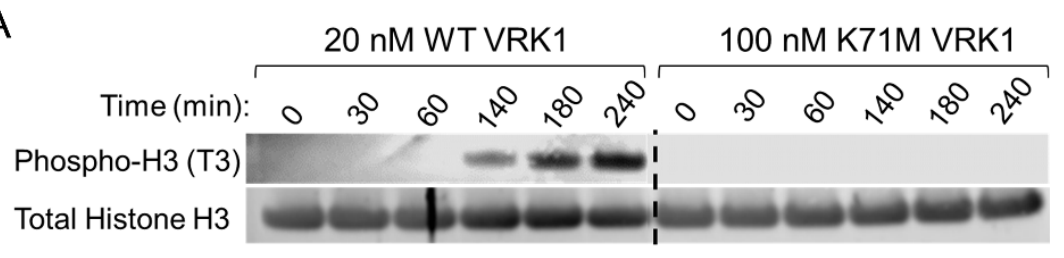

B

\begin{tabular}{ccccc}
\hline $\begin{array}{c}\text { Cell Line } \\
\text { Name }\end{array}$ & $\begin{array}{c}\text { VRK1 CRISPR } \\
\text { Score (CERES) }\end{array}$ & $\begin{array}{c}\text { VRK2 } \\
\text { Expression } \\
\text { log2(TPM+1) }\end{array}$ & $\begin{array}{c}\text { VRK2 } \\
\text { Methylation } \\
\text { Fraction }\end{array}$ & $\begin{array}{c}\text { MGMT } \\
\text { Methylation }\end{array}$ \\
\hline T98G & -0.72 & 4.26 & N/A & N/A \\
LN-229 & -0.59 & 3.92 & N/A & 0.77 \\
YH13 & -0.89 & 2.71 & 0.54 & 0.04 \\
KALS-1 & -1.05 & 2.91 & 0.18 & 0.14 \\
YKG1 & -0.30 & 4.12 & 0.01 & 0.05 \\
KS-1 & N/A & N/A & N/A & N/A \\
KNS60 & -0.81 & 0.37 & 0.94 & 0.35 \\
U-118 MG & -1.30 & 0.32 & 0.91 & 0.26 \\
SW 1088 & -1.35 & 0.26 & 0.85 & 0.62 \\
H4 & -0.59 & 0.16 & 0.97 & 0.37 \\
LN-18 & -1.02 & 0.15 & N/A & N/A \\
U-251 MG & -1.24 & 0.16 & 0.97 & 0.30 \\
\hline
\end{tabular}

Data source: DepMap Expression 21Q2 Public, CERES Score 21Q2, Fraction Methylation (1kb upstream TSS)

C

RKO

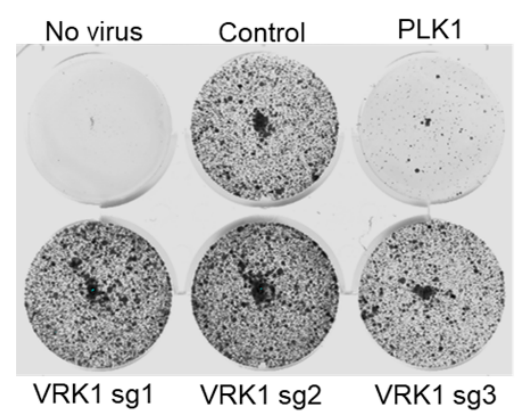

SNU-398

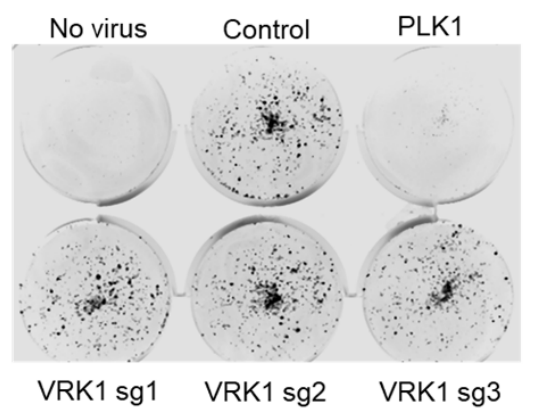

\section{Supplemental Figure 2.}

(A) Immunoblot depicting in vitro kinase activity of wildtype and mutant VRK1 on a histone H3 protein (B) Table showing CRISPR score, VRK2 expression, VRK2 methylation and MGMT methylation of a panel of GBM cell lines $(C)$ 14-day colony forming assay as in Figure 2A of VRK1 CRISPR knockdown in two non-GBM cell lines. 
A

LN229-dCas9-NTCi
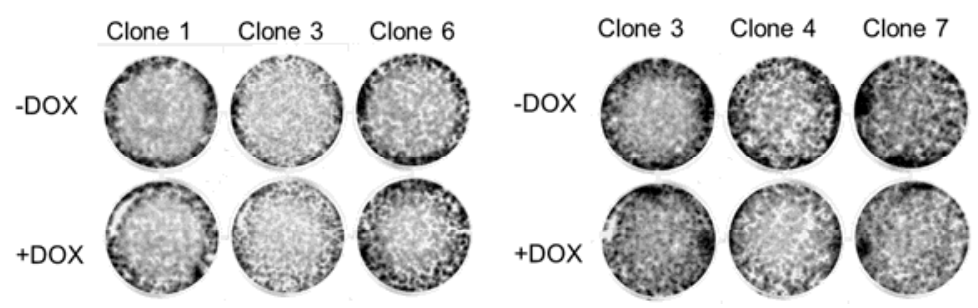

B

LN229-dCas9-

LN229-dCas9-

NTCguide-Clone 6

VRK1guide-Clone 7

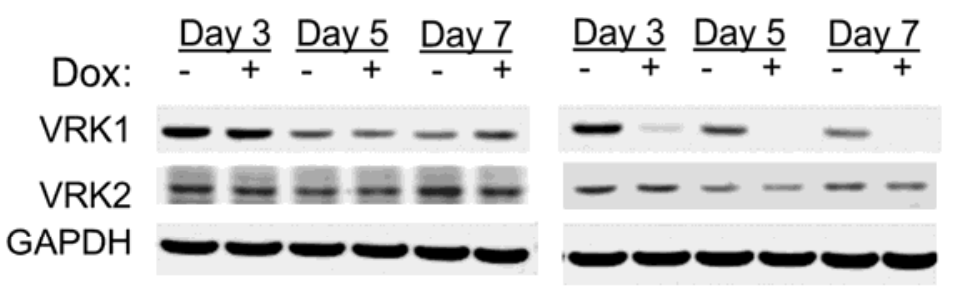

\section{Supplemental Figure 3.}

(A) 14-day colony forming assay of CRISPR-dCas9-KRAB inducible VRK1 knockdown (as in Figure 3A) of VRK2-high expressing LN229 clonal cell lines (B) Immunoblots of cells from $(A)$ demonstrating VRK1 knockdown. 
A
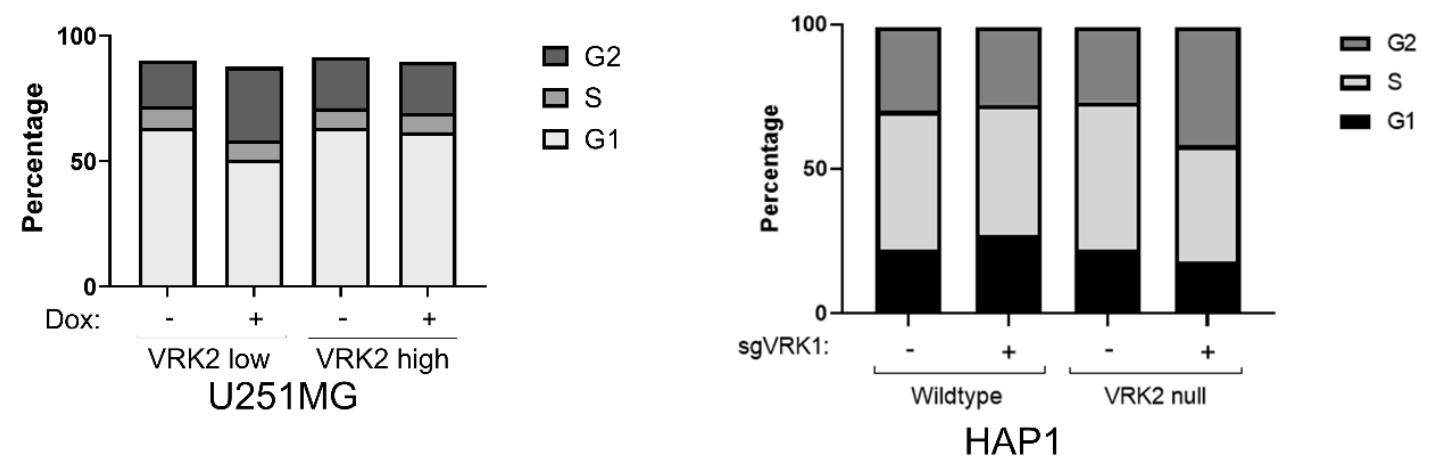

C
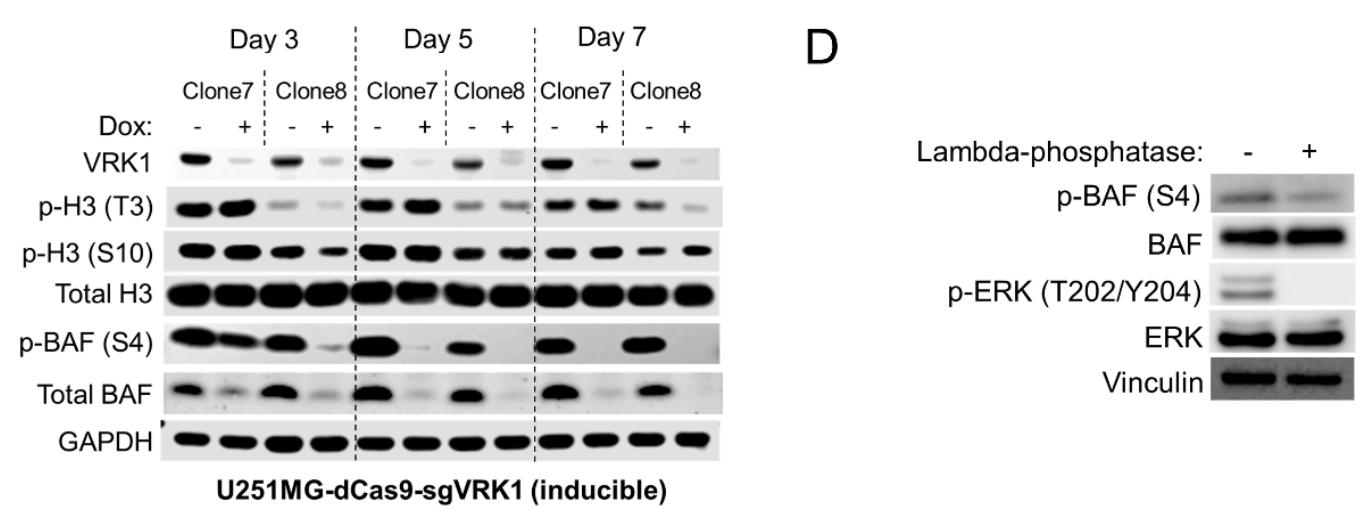

E Abnormal nuclear envelope

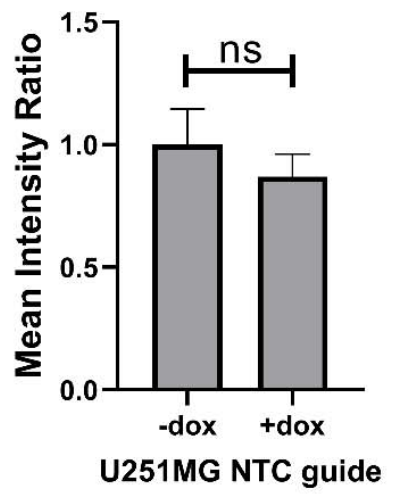

\section{Supplemental Figure 4.}

(A) Quantification of cell cycle distributions from Figure 4A (B) Quantification of cell cycle distributions from Figure 4B (C) Immunoblots of U251MG VRK2-low clonal cell lines at the indicated times (D) Immunoblots of HAP1 parental cell lysates treated with or without lambda phosphatase for 1 hour (E)Quantification of nuclear envelope phenotype in U251MG VRK2-low NTC control cell line treated with and without doxycycline. 


\section{Total proteomics}

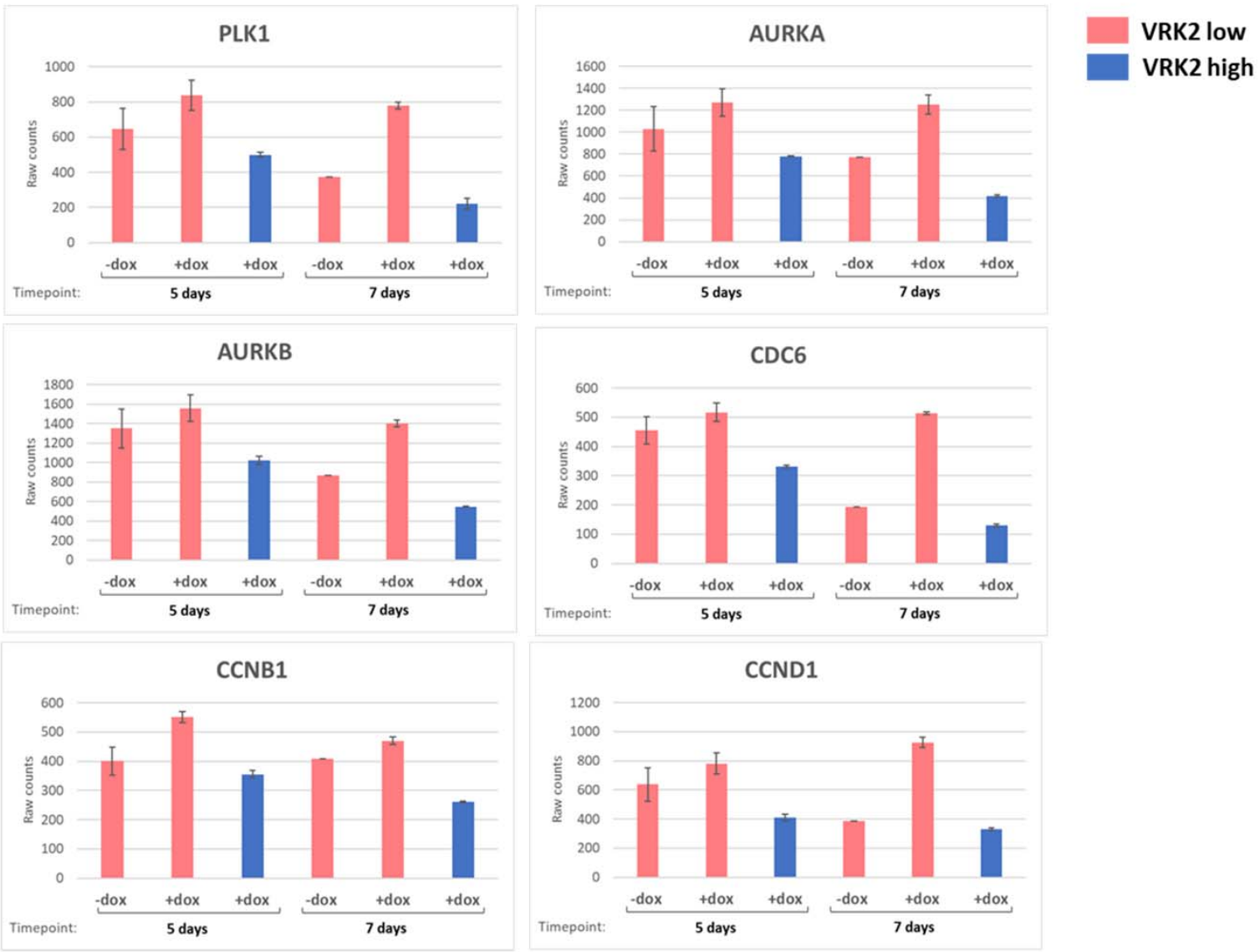

\section{Supplemental Figure 5.}

Total proteomics at 5- and 7-days post-doxycycline for cell cycle proteins. 


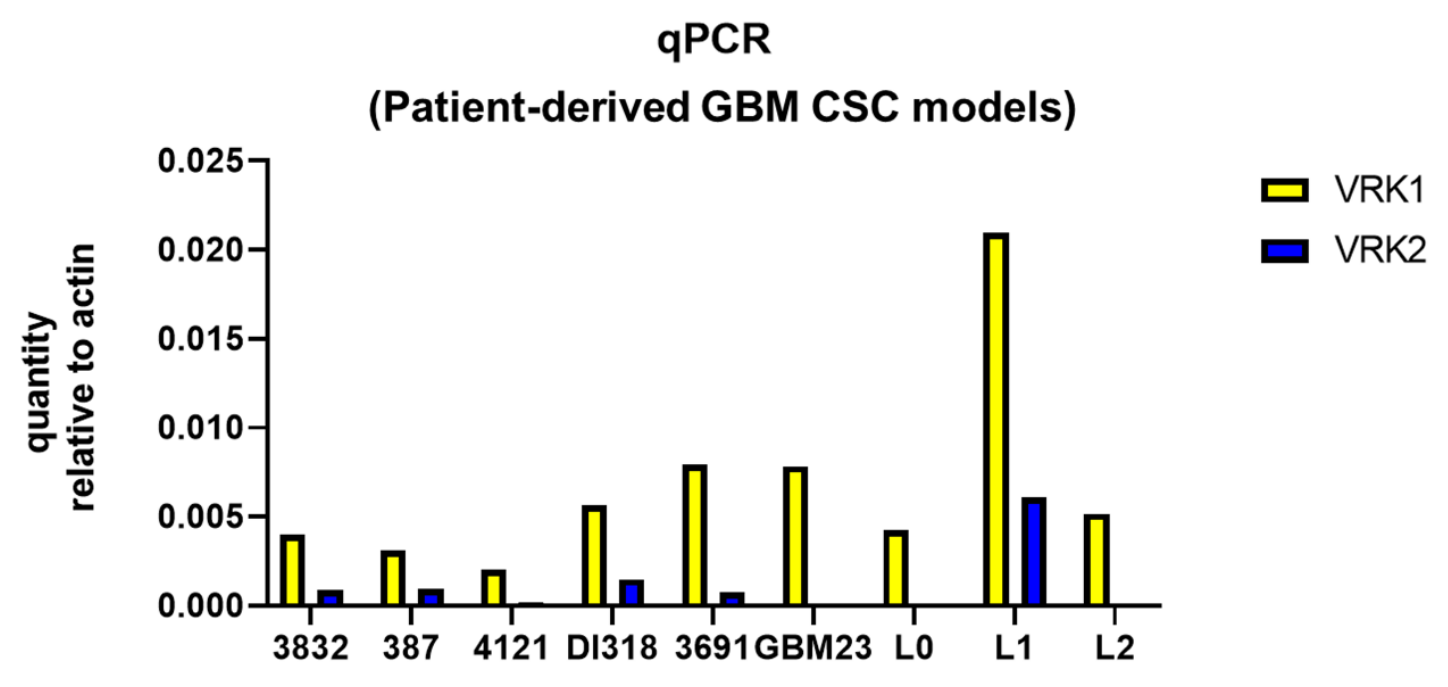

Supplemental Figure 6.

RT-qPCR for VRK1 and VRK2 from 9 CSC GBM models. 


\section{REFERENCES}

1. Huang A, Garraway LA, Ashworth A, Weber B. Synthetic lethality as an engine for cancer drug target discovery. Nat Rev Drug Discov. 2020;19:23-38.

2. Hartwell LH, Szankasi P, Roberts CJ, Murray AW, Friend SH. Integrating Genetic Approaches into the Discovery of Anticancer Drugs. Science. 1997;278:1064-8.

3. Kaelin WG. The Concept of Synthetic Lethality in the Context of Anticancer Therapy. Nat Rev Cancer. 2005;5:689-98.

4. Lord CJ, Ashworth A. PARP inhibitors: Synthetic lethality in the clinic. Science. 2017;355:1152-8.

5. Behan FM, lorio F, Picco G, Gonçalves E, Beaver CM, Migliardi G, et al. Prioritization of cancer therapeutic targets using CRISPR-Cas9 screens. Nature. 2019;568:511-6.

6. Neggers JE, Paolella BR, Asfaw A, Rothberg MV, Skipper TA, Yang A, et al. Synthetic Lethal Interaction between the ESCRT Paralog Enzymes VPS4A and VPS4B in Cancers Harboring Loss of Chromosome 18q or 16q. Cell Reports. 2020;33:108493.

7. Chan EM, Shibue T, McFarland J, Gaeta B, McPartlan JS, Ghandi M, et al. WRN Helicase is a Synthetic Lethal Target in Microsatellite Unstable Cancers. Biorxiv. 2018;502070.

8. Depmap B. DepMap 21Q1 Public. figshare. 2020;

9. Klerkx EPF, Lazo PA, Askjaer P. Review Emerging biological functions of the Vaccinia-Related Kinase (VRK) family. Histology and Histopathology. 2009;

10. Martín-Doncel E, Rojas AM, Cantarero L, Lazo PA. VRK1 functional insufficiency due to alterations in protein stability or kinase activity of human VRK1 pathogenic variants implicated in neuromotor syndromes. Sci Rep-uk. 2019;9:13381.

11. Valbuena A, Sanz-García M, López-Sánchez I, Vega FM, Lazo PA. Roles of VRK1 as a new player in the control of biological processes required for cell division. Cell Signal. 2011;23:1267-72.

12. Monsalve DM, Merced T, Fernández IF, Blanco S, Vázquez-Cedeira M, Lazo PA. Human VRK2 modulates apoptosis by interaction with $\mathrm{Bcl}-\mathrm{xL}$ and regulation of $\mathrm{BAX}$ gene expression. Cell Death Dis. 2013;4:e513-e513.

13. Blanco S, Klimcakova L, Vega FM, Lazo PA. The subcellular localization of vaccinia-related kinase-2 (VRK2) isoforms determines their different effect on p53 stability in tumour cell lines. Febs J. 2006;273:2487-504. 
14. Nichols RJ, Wiebe MS, Traktman P. The Vaccinia-related Kinases Phosphorylate the N'Terminus of BAF, Regulating Its Interaction with DNA and Its Retention in the Nucleus. Mol Biol Cell. 2006;17:2451-64.

15. Louis DN, Perry A, Wesseling P, Brat DJ, Cree IA, Figarella-Branger D, et al. The 2021 WHO Classification of Tumors of the Central Nervous System: a summary. Neurooncology. 2021;23:1231-51.

16. Parsons DW, Jones S, Zhang X, Lin JC-H, Leary RJ, Angenendt P, et al. An Integrated Genomic Analysis of Human Glioblastoma Multiforme. Science.

2008;321:1807-12.

17. Louis DN, Perry A, Reifenberger G, Deimling A von, Figarella-Branger D, Cavenee WK, et al. The 2016 World Health Organization Classification of Tumors of the Central Nervous System: a summary. Acta Neuropathol. 2016;131:803-20.

18. Kamson DO, Grossman SA. The Role of Temozolomide in Patients With Newly Diagnosed Wild-Type IDH, Unmethylated MGMTp Glioblastoma During the COVID-19 Pandemic. Jama Oncol. 2021;7:675-6.

19. Finch A, Solomou G, Wykes V, Pohl U, Bardella C, Watts C. Advances in Research of Adult Gliomas. Int J Mol Sci. 2021;22:924.

20. Stupp R, Hegi ME, Mason WP, Bent MJ van den, Taphoorn MJ, Janzer RC, et al. Effects of radiotherapy with concomitant and adjuvant temozolomide versus radiotherapy alone on survival in glioblastoma in a randomised phase III study: 5-year analysis of the EORTC-NCIC trial. Lancet Oncol. 2009;10:459-66.

21. Kammers K, Cole RN, Tiengwe C, Ruczinski I. Detecting significant changes in protein abundance. Eupa Open Proteom. 2015;7:11-9.

22. Meyers RM, Bryan JG, McFarland JM, Weir BA, Sizemore AE, Xu H, et al. Computational correction of copy number effect improves specificity of CRISPR-Cas9 essentiality screens in cancer cells. Nat Genet. 2017;49:1779-84.

23. Ghandi M, Huang FW, Jané-Valbuena J, Kryukov GV, Lo CC, McDonald ER, et al. Next-generation characterization of the Cancer Cell Line Encyclopedia. Nature. 2019;569:503-8.

24. Dempster JM, Rossen J, Kazachkova M, Pan J, Kugener G, Root DE, et al. Extracting Biological Insights from the Project Achilles Genome-Scale CRISPR Screens in Cancer Cell Lines. Biorxiv. 2019;720243.

25. Network CGAR, Brat DJ, Verhaak RGW, Aldape KD, Yung WKA, Salama SR, et al. Comprehensive, Integrative Genomic Analysis of Diffuse Lower-Grade Gliomas. New Engl J Medicine. 2015;372:2481-98. 
26. Brennan CW, Verhaak RGW, McKenna A, Campos B, Noushmehr H, Salama SR, et al. The Somatic Genomic Landscape of Glioblastoma. Cell. 2013;155:462-77.

27. Vega FM, Sevilla A, Lazo PA. p53 Stabilization and Accumulation Induced by Human Vaccinia-Related Kinase 1. Mol Cell Biol. 2004;24:10366-80.

28. Ren Z, Geng J, Xiong C, Li X, Li Y, Li J, et al. Downregulation of VRK1 reduces the expression of BANF1 and suppresses the proliferative and migratory activity of esophageal cancer cells. Oncol Lett. 2020;20:1163-70.

29. Molitor TP, Traktman P. Depletion of the protein kinase VRK1 disrupts nuclear envelope morphology and leads to BAF retention on mitotic chromosomes. Mol Biol Cell. 2014;25:891-903.

30. Jamin A, Wiebe MS. Barrier to Autointegration Factor (BANF1): interwoven roles in nuclear structure, genome integrity, innate immunity, stress responses and progeria. Curr Opin Cell Biol. 2015;34:61-8.

31. Samwer M, Schneider MWG, Hoefler R, Schmalhorst PS, Jude JG, Zuber J, et al. DNA Cross-Bridging Shapes a Single Nucleus from a Set of Mitotic Chromosomes. Cell. 2017;170:956-972.e23.

32. Kapanidou M, Curtis NL, Bolanos-Garcia VM. Cdc20: At the Crossroads between Chromosome Segregation and Mitotic Exit. Trends Biochem Sci. 2017;42:193-205.

33. Heald R, Mckeon F. Mutations of phosphorylation sites in lamin A that prevent nuclear lamina disassembly in mitosis. Cell. 1990;61:579-89.

34. Lee J, Kotliarova S, Kotliarov Y, Li A, Su Q, Donin NM, et al. Tumor stem cells derived from glioblastomas cultured in bFGF and EGF more closely mirror the phenotype and genotype of primary tumors than do serum-cultured cell lines. Cancer Cell. 2006;9:391-403.

35. Lathia JD, Mack SC, Mulkearns-Hubert EE, Valentim CLL, Rich JN. Cancer stem cells in glioblastoma. Gene Dev. 2015;29:1203-17.

36. Lathia JD, Gallagher J, Myers JT, Li M, Vasanji A, McLendon RE, et al. Direct In Vivo Evidence for Tumor Propagation by Glioblastoma Cancer Stem Cells. Plos One. 2011;6:e24807.

37. Adane B, Alexe G, Seong BKA, Lu D, Hwang EE, Hnisz D, et al. STAG2 loss rewires oncogenic and developmental programs to promote metastasis in Ewing sarcoma. Cancer Cell. 2021;39:827-844.e10. 
38. Gillani R, Seong BKA, Crowdis J, Conway JR, Dharia NV, Alimohamed S, et al. Gene Fusions Create Partner and Collateral Dependencies Essential to Cancer Cell Survival. Cancer Res. 2021;81:3971-84.

39. Sheffer M, Lowry E, Beelen N, Borah M, Amara SN-A, Mader CC, et al. Genomescale screens identify factors regulating tumor cell responses to natural killer cells. Nat Genet. 2021;53:1196-206.

40. Malone CF, Dharia NV, Kugener G, Forman AB, Rothberg MV, Abdusamad M, et al. Selective Modulation of a Pan-Essential Protein as a Therapeutic Strategy in Cancer. Cancer Discov. 2021;11:2282-99.

41. Helming KC, Wang X, Wilson BG, Vazquez F, Haswell JR, Manchester HE, et al. ARID1B is a specific vulnerability in ARID1A-mutant cancers. Nat Med. 2014;20:251-4.

42. Hoffman GR, Rahal R, Buxton F, Xiang K, McAllister G, Frias E, et al. Functional epigenetics approach identifies BRM/SMARCA2 as a critical synthetic lethal target in BRG1-deficient cancers. Proc National Acad Sci. 2014;111:3128-33.

43. Ogiwara H, Sasaki M, Mitachi T, Oike T, Higuchi S, Tominaga Y, et al. Targeting p300 Addiction in CBP-Deficient Cancers Causes Synthetic Lethality by Apoptotic Cell Death due to Abrogation of MYC Expression. Cancer Discov. 2016;6:430-45.

44. Couñago RM, Allerston CK, Savitsky P, Azevedo H, Godoi PH, Wells Cl, et al. Structural characterization of human Vaccinia-Related Kinases (VRK) bound to smallmolecule inhibitors identifies different P-loop conformations. Sci Rep-uk. 2017;7:7501.

45. Serafim RAM, Gama FH de S, Dutra LA, Reis CVD, Vasconcelos SNS, Santiago A da $S$, et al. Development of Pyridine-based Inhibitors for the Human Vaccinia-related Kinases 1 and 2. Acs Med Chem Lett. 2019;10:1266-71.

46. Santos CR, Rodríguez-Pinilla M, Vega FM, Rodríguez-Peralto JL, Blanco S, Sevilla A, et al. VRK1 Signaling Pathway in the Context of the Proliferation Phenotype in Head and Neck Squamous Cell Carcinoma. Mol Cancer Res. 2006;4:177-85.

47. Ben Z, Gong L, Qiu Y. High expression of VRK1 is related to poor prognosis in glioma. Pathology - Res Pract. 2018;214:112-8.

48. Li J, Wang T, Pei L, Jing J, Hu W, Sun T, et al. Expression of VRK1 and the downstream gene BANF1 in esophageal cancer. Biomed Pharmacother. 2017;89:108691.

49. Colmenero-Repiso A, Gómez-Muñoz MA, Rodríguez-Prieto I, Amador-Álvarez A, Henrich K-O, Pascual-Vaca D, et al. Identification of VRK1 as a New Neuroblastoma Tumor Progression Marker Regulating Cell Proliferation. Cancers. 2020;12:3465. 
50. Renbaum P, Kellerman E, Jaron R, Geiger D, Segel R, Lee M, et al. Spinal Muscular Atrophy with Pontocerebellar Hypoplasia Is Caused by a Mutation in the VRK1 Gene. Am J Hum Genetics. 2009;85:281-9.

51. Vinograd-Byk H, Sapir T, Cantarero L, Lazo PA, Zeligson S, Lev D, et al. The Spinal Muscular Atrophy with Pontocerebellar Hypoplasia Gene VRK1 Regulates Neuronal Migration through an Amyloid- Precursor Protein-Dependent Mechanism. J Neurosci. 2015;35:936-42.

52. Gonzaga-Jauregui C, Lotze T, Jamal L, Penney S, Campbell IM, Pehlivan D, et al. Mutations in VRK1 Associated With Complex Motor and Sensory Axonal Neuropathy Plus Microcephaly. Jama Neurol. 2013;70:1491-8.

53. Stoll M, Teoh H, Lee J, Reddel S, Zhu Y, Buckley M, et al. Novel motor phenotypes in patients with VRK1 mutations without pontocerebellar hypoplasia. Neurology. 2016;87:65-70.

54. Vinograd-Byk H, Renbaum P, Levy-Lahad E. Vrk1 partial Knockdown in Mice Results in Reduced Brain Weight and Mild Motor Dysfunction, and Indicates Neuronal VRK1 Target Pathways. Sci Rep-uk. 2018;8:11265.

55. Campillo-Marcos I, García-González R, Navarro-Carrasco E, Lazo PA. The human VRK1 chromatin kinase in cancer biology. Cancer Lett. 2021;503:117-28.

56. Furukawa K, Sugiyama S, Osouda S, Goto H, Inagaki M, Horigome T, et al. Barrierto-autointegration factor plays crucial roles in cell cycle progression and nuclear organization in Drosophila. J Cell Sci. 2003;116:3811-23.

57. Gorjánácz M, Klerkx EP, Galy V, Santarella R, López-Iglesias C, Askjaer P, et al. Caenorhabditis elegans BAF-1 and its kinase VRK-1 participate directly in post-mitotic nuclear envelope assembly. Embo J. 2007;26:132-43.

58. Margalit A, Segura-Totten M, Gruenbaum Y, Wilson KL. Barrier-to-autointegration factor is required to segregate and enclose chromosomes within the nuclear envelope and assemble the nuclear lamina. P Natl Acad Sci Usa. 2005;102:3290-5.

59. Zheng R, Ghirlando R, Lee MS, Mizuuchi K, Krause M, Craigie R. Barrier-toautointegration factor (BAF) bridges DNA in a discrete, higher-order nucleoprotein complex. Proc National Acad Sci. 2000;97:8997-9002.

60. Jamin A, Wiebe MS. Barrier to Autointegration Factor (BANF1): interwoven roles in nuclear structure, genome integrity, innate immunity, stress responses and progeria. Curr Opin Cell Biol. 2015;34:61-8. 
61. Puente XS, Quesada V, Osorio FG, Cabanillas R, Cadiñanos J, Fraile JM, et al. Exome sequencing and functional analysis identifies BANF1 mutation as the cause of a hereditary progeroid syndrome. Am J Hum Genet. 2011;88:650-6.

62. Burla R, Torre ML, Merigliano C, Vernì F, Saggio I. Genomic instability and DNA replication defects in progeroid syndromes. Nucl Austin Tex. 2018;9:368-79.

63. Guey B, Wischnewski M, Decout A, Makasheva K, Kaynak M, Sakar MS, et al. BAF restricts cGAS on nuclear DNA to prevent innate immune activation. Science. 2020;369:823-8.

64. Rhun EL, Preusser $M$, Roth $P$, Reardon DA, Bent $M$ van den, Wen $P$, et al. Molecular targeted therapy of glioblastoma. Cancer Treat Rev. 2019;80:101896.

65. Navarro-Carrasco E, Lazo PA. VRK1 Depletion Facilitates the Synthetic Lethality of Temozolomide and Olaparib in Glioblastoma Cells. Frontiers Cell Dev Biology. $2021 ; 9: 683038$. 